\title{
Gatekeeper helix activates Golgi SM protein Sly1 and directly mediates close-range vesicle tethering
}

\author{
M. Duan ${ }^{1^{\star}}$, R.L. Plemel ${ }^{1^{\star}}$, T. Takenaka ${ }^{1,5}$, A. Lin $^{6}$, B.M. Delgado ${ }^{6}$, U. \\ Nattermann ${ }^{1,3,4}$, D.P. Nickerson ${ }^{6}$, J. Mima ${ }^{7}$, E.A. Miller ${ }^{8}$, A.J. Merz ${ }^{1,2,5}$
}

Departments of ${ }^{1}$ Biochemistry; ${ }^{2}$ Physiology \& Biophysics; ${ }^{3}$ Biophysics, Structure, and Design Graduate Program; and ${ }^{4}$ Institute for Protein Design, University of Washington, Seattle, United States, 98195-7350

${ }^{5}$ Tokyo Institute of Technology, Tokyo, Japan

${ }^{6}$ Department of Biology, California State University, San Bernardino, United States

${ }^{7}$ Institute for Protein Research, Osaka University, Osaka, Japan

${ }^{8} \mathrm{MRC}$ Laboratory of Molecular Biology, Cambridge, England

${ }^{\star}$ Equal contributor

§Corresponding author: merza@uw.edu·+1-206-616-8308 


\section{ABSTRACT (c. 160 words)}

The essential Golgi protein Sly1 is a member of the SM (Sec1/mammalian Unc-18) family of SNARE chaperones. Sly1 was originally identified through gain-of-function alleles that bypass requirements for diverse vesicle tethering factors. Employing genetic analyses and chemically defined reconstitutions of ER-Golgi fusion, we discovered that a loop conserved among Sly1 family members is not only autoinhibitory, but also acts as a positive effector. An amphipathic helix within the loop directly binds high-curvature membranes; membrane binding is required for relief of Sly1 autoinhibition and allows Sly1 to directly tether incoming vesicles to the QaSNARE on the target organelle. The SLY1-20 allele bypasses requirements for diverse tethering factors but loses this functionality if Sly1 membrane binding is impaired. We propose that long-range tethers, including Golgins and multisubunit tethering complexes, hand off vesicles to Sly1, which then tethers at close range to activate SNARE assembly and fusion in the early secretory pathway.

\section{INTRODUCTION}

Traffic through the secretory and endocytic systems depends on accurate and timely targeting of transport vesicles to acceptor organelles. The terminal stage of targeting is membrane fusion, catalyzed by the formation of trans-SNARE complexes that zipper together, doing the mechanical work of moving two membranes into proximity and driving their merger. Although SNAREs alone can drive fusion and confer some compartmental selectivity, spontaneous SNARE assembly is slow and error-prone. Consequently, an array of tethering factors and SNARE chaperones are indispensable in vivo (Baker and Hughson, 2016; Gillingham and Munro, 2019). For example, every SNARE-mediated fusion event that has been closely examined requires a cofactor of the Sec1/mammalian Unc-18 (SM) family.

For decades the mechanisms of SM protein function were enigmatic (Carr and Rizo, 2010; Rizo and Sudhof, 2012; Sudhof and Rothman, 2009) but biochemical work, structural studies, and single-molecule force spectroscopy suggest that SM proteins are assembly chaperones for trans-SNARE complex formation, and that SMs act, at least in part, by 
templating the initial SNARE zippering reaction (Baker et al., 2015; Jiao et al., 2018) and by disassembly proteins Sec17/ $\alpha$-SNAP and Sec18/NSF (Lobingier et al., 2014; Ma et al., 2013; Schwartz et al., 2017; Xu et al., 2010). There are four subfamilies of SM proteins. The budding yeast Saccharomyces cerevisiae has one representative of each. Vps33, the first SM identified genetically, controls fusion at late endosomes and lysosomes (Banta et al., 1990; Patterson, 1932; Sevrioukov et al., 1999). Vps45 controls fusion at early endosomal compartments (Cowles et al., 1994; Piper et al., 1994). Sec1 and its orthologs (Unc-18/Munc-18) control exocytosis (Grote et al., 2000; Novick et al., 1979; Verhage et al., 2000; Wu et al., 1998). Finally, fusion at the Golgi, and probably at the endoplasmic reticulum (ER), is controlled by Sly1 (Li et al., 2005; Lupashin et al., 1996; Ossig et al., 1991; Peng and Gallwitz, 2002; Sogaard et al., 1994).

The genetics of $S L Y 1$ are complex and revealing. Ypt1 (yeast Rab1) is an essential regulator of docking and fusion at the Golgi. $S L Y 1$ was originally identified through an allele,

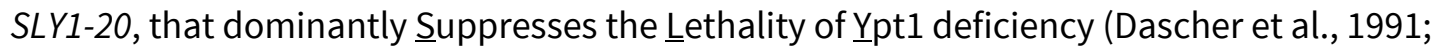
Ossig et al., 1991; Ossig et al., 1995). Subsequent work by several groups showed that SLY1-20 suppresses deficiencies not only of Ypt1, but of numerous other factors that promote ER and Golgi traffic. These include the Dsl complex (ds/1 was originally identified through its genetic interaction with SLY1-20; Reilly et al., 2001; Vanrheenen et al., 2001); the COG complex (cog2, cog3; VanRheenen et al., 1998; VanRheenen et al., 1999); the TRAPP complexes (bet3-1; Sacher et al., 1998); the Golgin coiled-coil tether Uso1 (yeast p115; Sapperstein et al., 1996); Ypt6

50 (yeast Rab6) and its nucleotide exchange complex (ric1; Bensen et al., 2001; Li et al., 2007); and the Ypt6 effector complex GARP (vps53; Vanrheenen et al., 2001). In addition, SLY1-20 suppresses partial deficiencies of Golgi SNAREs (sec22; Ossig et al., 1991); COPI coat subunits sec21; (Ossig et al., 1991); and the COPI Arf GAP Glo3 (Vanrheenen et al., 2001).

SLY1-20 and the similar allele SLY1-15 encode missense substitutions at adjacent positions within a loop insertion that is evolutionarily conserved among Sly1 subfamily members, but absent from the other three SM subfamilies (Dascher et al., 1991; Li et al., 2007). On this basis it was hypothesized that the Sly1 loop is auto-inhibitory, and that SLY1-20 and related alleles gain function by releasing the loop from its closed, autoinhibitory state 
(Bracher and Weissenhorn, 2002; Li et al., 2007). This proposal was strengthened by the discovery that the Sly1 loop occludes a conserved site which, in the lysosomal SM Vps33, binds R/v-SNAREs with high affinity (Baker et al., 2015).

The physiological mechanism by which the Sly1 loop's putative auto-inhibitory activity is released to promote SNARE complex formation is unknown, but was suggested to require Ypt1, the yeast Rab1 ortholog (Bracher and Weissenhorn, 2002; Li et al., 2007). Here, we show that the loop's inhibitory activity is released when an amphipathic helix within the loop interacts directly with the incoming vesicle membrane's lipid bilayer. Moreover, the loop allows Sly1 to directly tether incoming vesicles: the Sly $1 \mathrm{~N}$-lobe is anchored to the Qa/t-SNARE on the target organelle, while the Sly1 regulatory loop binds the vesicle lipid bilayer. We propose that this membrane binding steers Sly1 into an orientation optimal for productive R/v-SNARE association and trans-complex assembly. This schema explains how Sly1-20 can bypass the otherwise essential functions of so many different Golgi tethering factors, and suggests that the Sly1 regulatory loop links Sly1 activation, the capture of transport vesicles addressed to organelles of the early secretory pathway, and productive trans-SNARE complex assembly. 


\section{RESULTS}

\section{New SLY1 alleles define a regulatory loop in Sly1}

We thought it likely that early screens which identified $S L Y 1^{*}$ bypass alleles were not saturated, and that a more focused screen might yield additional informative alleles. Uso1 is a Golgin-class tether that is a direct effector of Ypt1/Rab1. Loss of Uso1 is lethal, and this lethality is suppressed by SLY1-20 (Ballew et al., 2005; Sapperstein et al., 1996). We therefore designed a selection for dominant $S L Y 1^{*}$ alleles that could suppress the loss of USO1 (Fig. 1A). (In this report, sets of $S L Y 1$ alleles and their products are referred to collectively as $S L Y 1^{\star}$ and Sly ${ }^{\star}$.) Our screen retrieved many $S L Y 1^{*}$ alleles, most carrying multiple missense substitutions. From these, individual missense substitutions were re-introduced into wild type SLY1 and tested for their ability to suppress deficiencies of Uso1 or Ypt1 (Fig. 1B;

Supplementary Table 1). Importantly, our screen retrieved the original SLY1-20 and SLY1-15 alleles. We also identified suppressing substitutions at nearby sites on helix $\alpha 20$, and on the short segment linking helices $\alpha 20$ and $\alpha 21$. Additionally, we identified suppressing substitutions at the base of the Sly1-specific loop, and at positions cradling the base of the
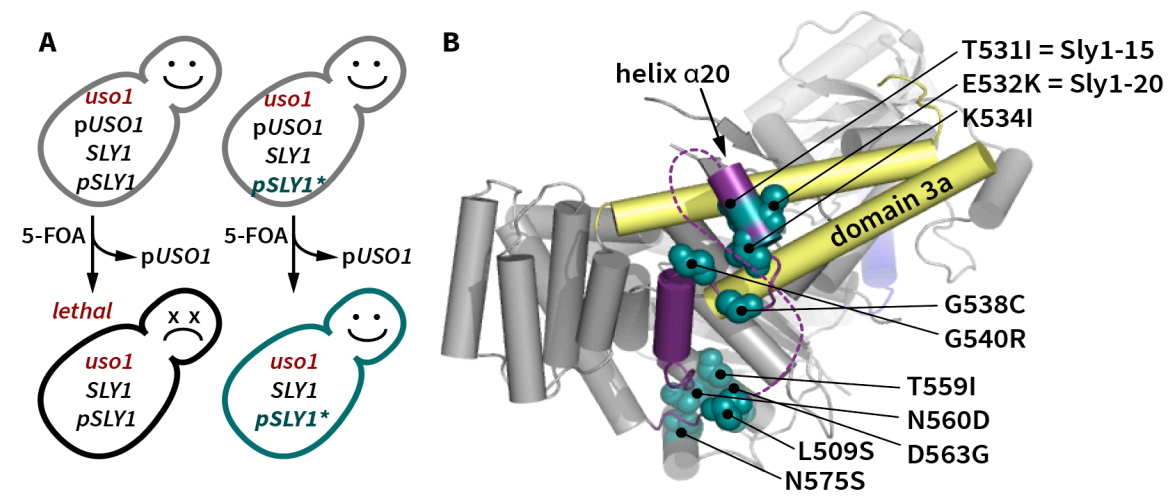

Fig. 1. New gain-of-function $S L Y 1$ alleles. A. Selection used in this study. A library of $S L Y 1^{*}$ alleles was constructed by PCR-based mutagenesis and cloned into a single-copy plasmid. The library was then transformed into a SLY1 uso1D strain, with USO1 provided on a balancer plasmid bearing the counterselectable URA3 marker. Ejection of pUSO1 was forced by 5-fluoroorotic acid (5-FOA). This positively selects viable cells carrying dominant mutant $S L Y 1^{*}$ alleles that bypass the otherwise essential USO1 requirement. B. Locations within Sly1 (PDB: 1MQS) of single missense substitutions that suppress requirements for Ypt1, or for both Ypt1 and Uso1. The loop is indicated in purple, with the dashed line denoting the portion of the loop not resolved in the structure. Yellow shading indicates the domain $3 a$ helical hairpin which, by analogy to Vps33 and Munc18-1, is hypothesized to scaffold assembly of Qa- and R-SNARE trans-complexes. 
loop, but non-adjacent within the linear polypeptide sequence. One of these was T559I. A genomic survey for gene pairs exhibiting spontaneous suppressing interactions found that a substitution at the same position, T559K, dominantly suppressed deficiencies of both the GARP subunit Vps53 and the Arf GAP Glo3 (van Leeuwen et al., 2016). Most of the gain-offunction single substitutions that we tested suppressed ypt1-3 but, in contrast to the multisite mutants obtained in the initial selection for uso1 $\Delta$ bypass, were unable to suppress uso1 $\Delta$. (Supplementary Fig. S1; Supplementary Table 1). Thus, strong Sly1 gain-of-function phenotypes can arise through individual substitutions or through the compounded effects of multiple weak driver substitutions. These results show that earlier screens were not, as had been suggested, saturated (Li et al., 2007).

As noted by Baker and coworkers (2015), helices $\alpha 20$ and $\alpha 21$ sit atop two conserved regions that in Vps33 are of special importance for SNARE binding: domain 3a, which serves as a scaffold to nucleate the parallel, in-register assembly of the Qa- and R-SNAREs, and an aromatic pocket that serves as a high-affinity anchoring point for the R-SNARE juxtamembrane linker. On the basis of the Vps33 structures and the original SLY1-20 and SLY115 alleles, Baker et al. (2015) speculated that when closed, the Sly1 loop might prevent RSNARE binding to Sly1. The dominant suppressors obtained in our screen and data presented below reinforce and extend that model.

\section{Sly1 bypass suppressors are hyperactive in a minimal fusion system}

In vivo genetic tests and crude in vitro transport systems (Baker et al., 1988; Ballew et al., 2005; Ruohola et al., 1988) cannot tell us whether Sly1* mutants must interact with additional proteins beyond the core SNARE fusion machinery to manifest gain of function. To overcome this limitation, we developed a chemically defined reconstituted proteoliposome (RPL) system to monitor fusion driven by ER-Golgi SNAREs (Fig. 2A). This system, adapted from an assay developed to study homotypic vacuole fusion (Zucchi and Zick, 2011), employs two orthogonal pairs of Förster resonance energy transfer (FRET) probes, to simultaneously monitor both lipid and content mixing in small $(20 \mu \mathrm{L})$ reaction volumes. Although we present 
only content mixing data in this manuscript, the lipid mixing signal provides an intrinsic

(2)

control, allowing us to detect partial hemifuison or fusion that is accompanied by lysis.

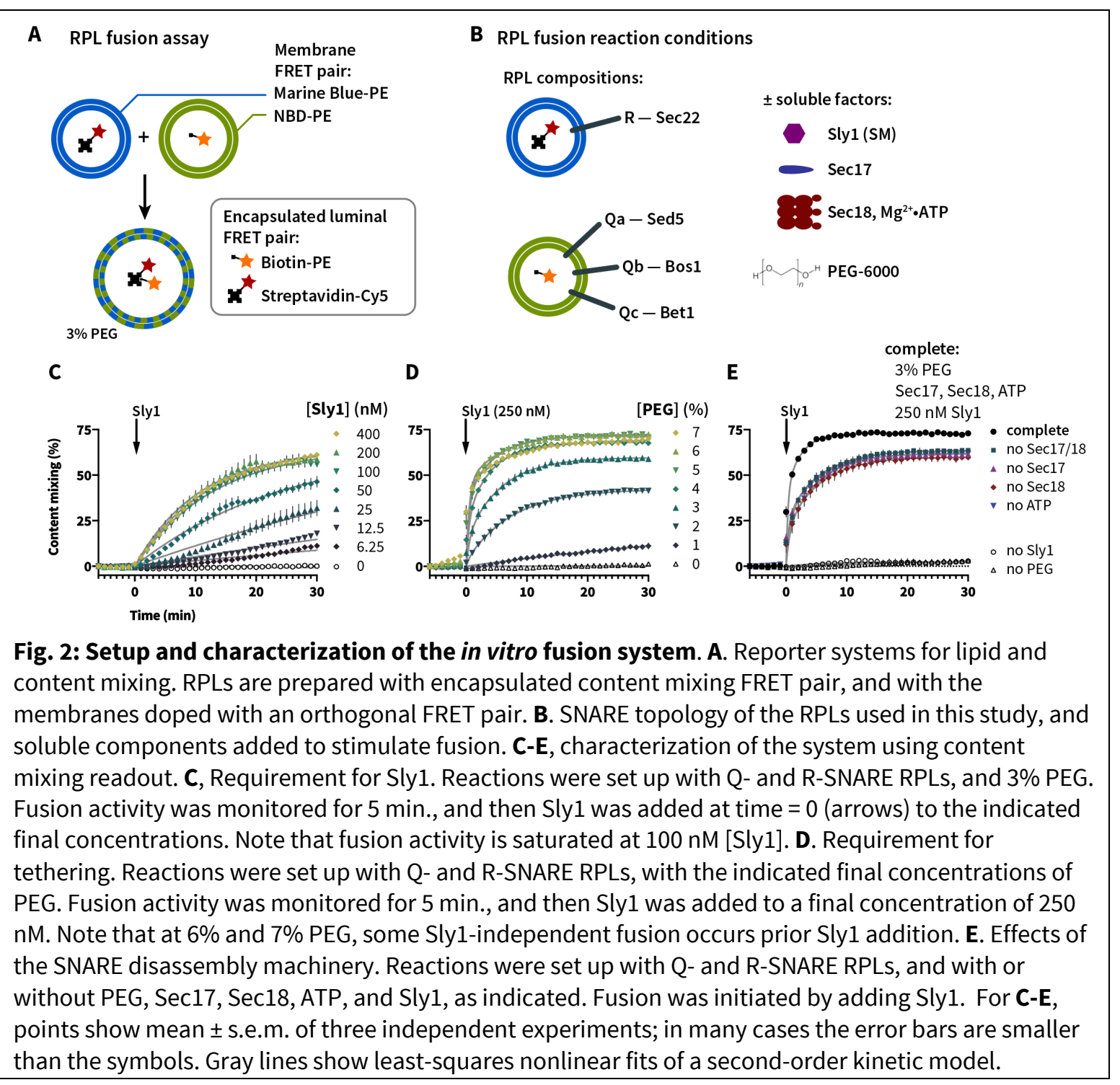

In previous work SMs were shown to stimulate SNARE-mediated lipid mixing, but only in the presence of tethering factors or molecular crowding agents that substitute for tethering factors (Furukawa and Mima, 2014; Yu et al., 2015). Consistent with these previous studies, content mixing in heterotypic reactions between RPLs bearing the R-SNARE Sec22, and RPLS bearing the Q-SNAREs Sed5, Bos1 and Bet1, was strongly stimulated only when both Sly1 and a crowding agent (polyethylene glycol 6000; PEG) were provided (Fig. 2C,D). Under these experimental conditions the stimulatory effect of Sly1 saturated at $100 \mathrm{nM}$. Two other studies 
have reported in vitro stimulation of fusion by Sly 1 , though at a $45 \times$ higher concentration of Sly1 than the $100 \mathrm{nM}$ used in most of our experiments (Furukawa and Mima, 2014; Jun and Wickner, 2019). Pre-incubation of the RPLs with $\mathrm{Mg}^{2+}$.ATP and the SNARE disassembly chaperones Sec17 and Sec18 (yeast $\alpha$-SNAP and NSF) resulted in immediate and almost complete fusion upon Sly1 addition (Fig. 2E), likely indicating that SNAREs on the RPLS equilibrate between productive and refractory configurations, and that Sec17/18-mediated disassembly shifts this equilibrium toward productive, Sly1-reactive configurations.

Next, we compared the activity of wild type Sly1 to three bypass suppressors: Sly1-20 and two of the new alleles identified in our screen. Each variant was tested in reactions containing 3\% or $0 \%$ polyethylene glycol-6000 (PEG). At 3\% PEG all four Sly1 variants drove fusion with similar efficiency (Fig. 3A). In marked contrast, at 0\% PEG (Fig. 3B) all three Sly1 suppressor mutants drove fusion substantially more efficiently than the wild type. In reactions containing Sec17, Sec18, and $\mathrm{Mg}^{2+}$ ATP (Fig. 3C,D) the same overall pattern emerged. As PEG bypasses requirements for tethering factors and potentiates SM-mediated fusion, our results show for the first time that these Sly1 gain-of-function mutants are intrinsically hyperactive, requiring only SNAREs (or SNAREs and disassembly chaperones) to stimulate fusion, and not additional cellular factors such as Rabs or tethering factors. These results directly mirror the in vivo genetic suppression patterns observed for $S L Y 1-20$ and otherwise essential vesicle tethering regulators and effectors. 


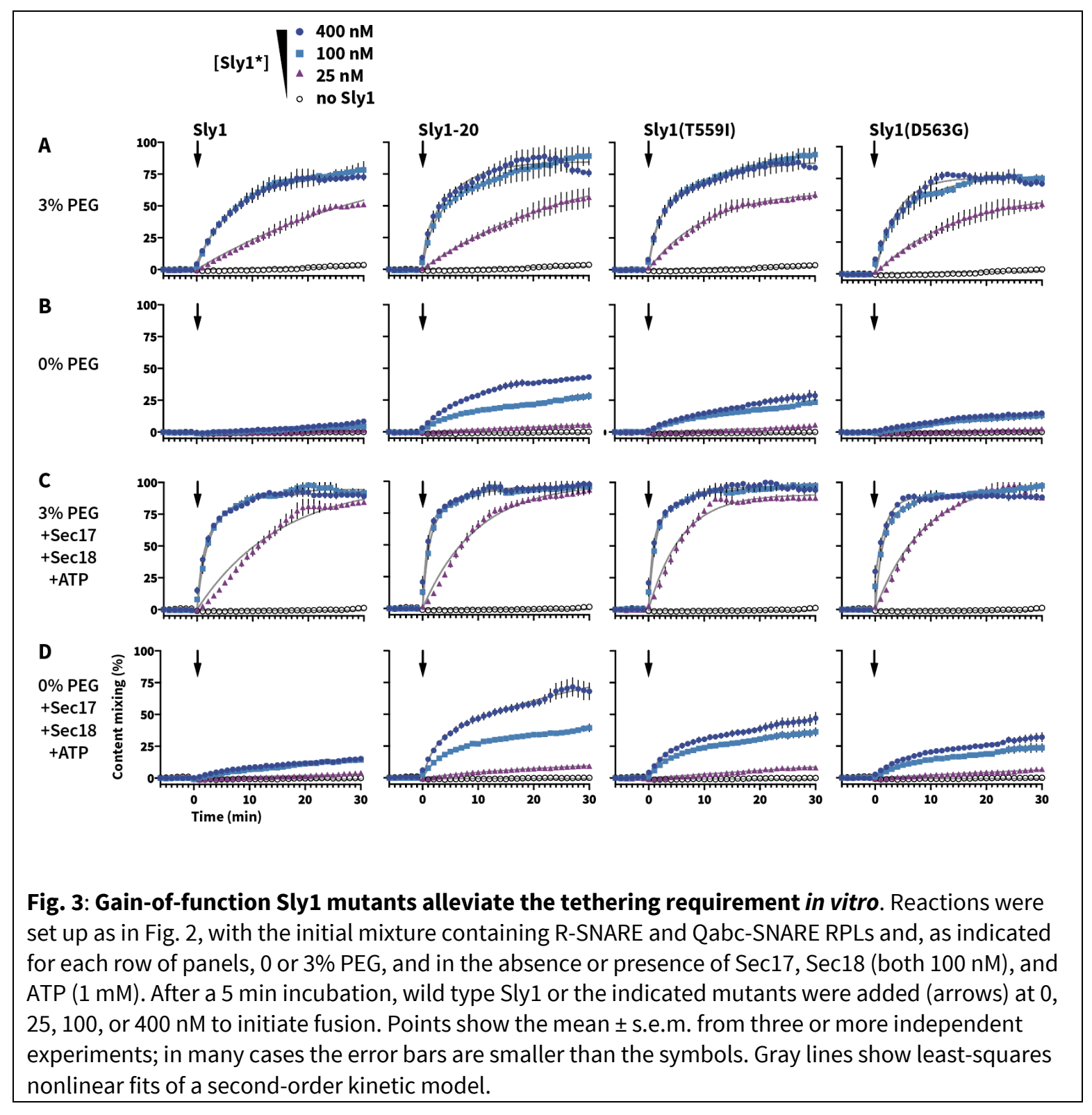




\section{The Sly1 regulatory loop has positive as well as negative functions}

If the Sly1 loop is autoinhibitory, we might predict that excision of the entire loop should hyperactivate Sly1 as much as or more than the suppressing mutations characterized above. To test this hypothesis, we used the ROSETTA software environment (Leaver-Fay et al., 2011) to design a set of Sly1 variants in which the loop is replaced by short peptide linkers (Fig. 4A; Supplementary Table 2). Surprisingly, each of the "loopless" sly1 mutants tested in vivo exhibited recessive lethality or slow growth in the presence of the counterselection agent 5FOA at $30^{\circ} \mathrm{C}$; temperature sensitivity; and an inability to bypass deficiencies in YPT1 or USO1. One mutant, sly1-0_2, exhibited somewhat more robust growth compared to the other alleles when present as the sole copy of SLY1. sly1-0_2 was therefore called sly1 1 loop and subjected to further scrutiny.

To gain genome-scale insight into the sly14loop allele's loss of function, we used synthetic genome array (SGA) analysis. SGA measures the synthetic sickness or rescue (suppression) of a query allele versus a genome-scale collection of loss-of-function alleles (Tong and Boone, 2005). sly1 1 loop was knocked in at the genomic SLY1 locus. The resulting strain grew normally on rich YPD medium containing 5-FOA at $30^{\circ} \mathrm{C}$, but slowly compared to strains with wild-type or hyperactive $S L Y 1$ alleles at $37^{\circ} \mathrm{C}$ (Fig. 4B). When subjected to SGA analysis, sly14loop had a synthetic-sick or synthetic-lethal interaction with ten of twelve genes previously reported to exhibit positive suppressing interactions with $S L Y 1-20$, as well as with dozens of additional genes that function in organelle biogenesis and membrane traffic especially traffic into and through the Golgi (Fig. 4C; Supplementary Dataset 1). Gene Ontology (GO) analysis (Mi et al., 2019; The Gene Ontology Consortium, 2019) recvealed that enrichment for these gene functions was both selective and statistically significant (Fig. 4D). Wild-type SLY1 activity is also required for resistance to the toxic effects of SEC17 overproduction (Lobingier et al., 2014; Schwartz et al., 2017), and SEC17 overproduction caused a severe recessive growth defect in sly1 1 loop cells (Fig. 4E). Overexpressino of a mutant, sec17-FSMS, caused an even more severe growth defect. Together, the genetic and functional genomic results show that sly $1 \Delta l o o p$ is a recessive loss-of-function allele and not, as expected, a dominant suppressor. 
A

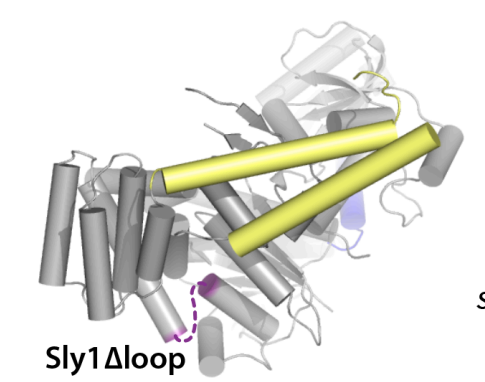

C Rabs

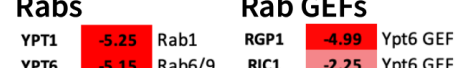

\begin{tabular}{l|llllll}
\hline YPT6 & -5.15 & Rab6/9 & RIC1 & $-\mathbf{2 . 2 5}$ & Ypt6 GEF \\
\hline
\end{tabular}

$\begin{array}{lll}\text { VPS21 } & -0.85 & \text { Rab5 } \\ \text { YPT } & -0.42 & \text { Rab7 }\end{array}$

\begin{tabular}{c|c|c} 
YPT & -0.42 & $R a b 7$ \\
YPT11 & -0.20 & \\
YPT31 & -0.09 & Rab11
\end{tabular}

$\begin{array}{lll}\text { YPT31 } & -0.09 & \text { Rab11 }\end{array}$

$\begin{array}{lll}\text { YPT53 } & -0.09 & \text { Rab5 }\end{array}$

$\begin{array}{lll}\text { YPT52 } & -0.01 & \text { Rab5 }\end{array}$

$\begin{array}{lll}\text { YPT32 } & 0.01 & \text { Rab11 }\end{array}$

\begin{tabular}{l|l|l|} 
SEC4 & 0.11 & Rab3
\end{tabular}

\begin{tabular}{l|l|l} 
YPT10 & 0.40 \\
\hline
\end{tabular}

Rab GAPs

GYP1 -4.73 Ypt1 GAP

GYP8 -0.32

GYL1 -0.06

GYP7 0.04

GYP6 0.11

GYP5 0.12

SEC2 -1.65 Sec4 GEF

$\begin{array}{lll}\text { VPS9 } & -0.96 & \text { Rab5 GEF }\end{array}$

\begin{tabular}{l|l|l} 
MON1 & 0.11 & Ypt7 GEF
\end{tabular}

\begin{tabular}{l|l|l} 
CCZ1 & -0.27 & Ypt7 GEF
\end{tabular}

TRAPP

\begin{tabular}{l|l|l}
\hline TRS20 & -5.19 & core \\
\hline BET3 & -2.09 & core
\end{tabular}

\begin{tabular}{l|l|l} 
BET3 & -2.09 & core \\
BET5 & -1.99 & core \\
\hline
\end{tabular}

TRS85 $\quad-0.95$ TRAPP III (Ypt1 GE

\begin{tabular}{|l|l|l|}
\hline TRS85 & -0.95 & TRAPP \\
\hline TRS31 & -0.36 & core \\
\hline
\end{tabular}

TRS23 -0.27 core

\begin{tabular}{l|l|l}
\hline TRS65 & -0.17 & TRAPP \\
\hline TRS33 & -0.05 & core
\end{tabular}

$\begin{array}{lll}\text { TRS130 } & 0.73 & \text { TRAPP II (Ypt31/32 GEF) }\end{array}$

\begin{tabular}{l|ll} 
TRS120 & 1.27 & TRAPP $\|$ (Ypt31/32 GEF)
\end{tabular}

\section{B}

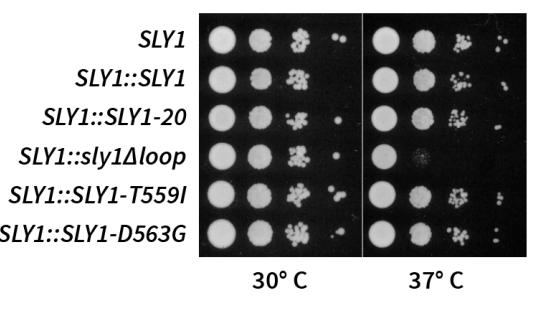

D

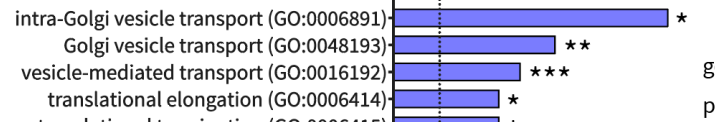

translationalengation $(G 0: 0004$

translational termination (GO:0006415) translation initiation complex (GO:0001677) translation (GO:0006412) cellular localization (GO:0051641) transport (GO:0006810) establishment of localization (GO:0051234) cellular component organization (GO:0016043) localization (GO:0051179)
ne expression (GO:0010467) gene expression (GO:0010467)
Unclassified (UNCLASSIFIED)

COG

COG5

COG7

$\cos 4-0.21$

\begin{tabular}{l|l} 
COG2 & 0.07
\end{tabular}

GARP Qa-SNARE

VPS53

VPS51 -2.20

Dsl

TIP20 -1.03

\begin{tabular}{c|r|}
\hline DSL1 & -0.29 \\
\hline SEC39 & -0.04
\end{tabular}

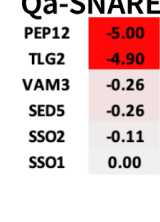

COPI

\begin{tabular}{l|l|}
\hline SEC28 & -4.66 \\
\hline SEC27 & -4.06 \\
\hline
\end{tabular}

RET3 -3.84

SEC26 -0.81

RET1 -0.15

COPII

SAR1 -4.24 GTPase

SEC12 $-\mathbf{0 . 2 0}$ Sar1 GEF

$\begin{array}{llll}\text { SEC16 } & -0.16 & \text { ER exit }\end{array}$

$\begin{array}{lll}\text { SFB2 } & -\mathbf{0 . 9 7} & \text { Sec24 paralog }\end{array}$

GOT1 -5.09 accessory

\begin{tabular}{l|ll} 
ERV14 & -3.14 & accessory
\end{tabular}

ERV25 -0.64 accessory

\section{E}

genome: sly1 $\Delta$

pRS416::SLY1 (URA3)

*

pRS41

(6)

SLY1 |

- pDN524 (control)

$+5-$ FOA

pDN524::SEC17

pDN524::sec17-FSMS

Fold enrichment

Fig. 4. The Sly1 regulatory loop has a positive function in vivo. A, Diagram showing the location of Sly1 loop replacement with short synthetic linker regions (dashed line; model derived from PDB $1 M Q S)$. Sequences of the linker insert designs, and growth phenotypes of the corresponding mutants, are presented in Supplementary Table 2. The domain 3a SNARE assembly template is shown in yellow. B, The sly $1 \Delta$ loop mutant is temperature sensitive for growth. Dilutions of liquid cultures were spotted as $10 x$ serial dilutions onto YPD agar plates and incubated for 2 days at $30^{\circ}$ or $37^{\circ} \mathrm{C}$. These are knock-ins at the genomic SLY1 locus, in the Y8205 strain background used for SGA analysis. C, Selected SGA results. Genes exhibiting synthetic interactions with sly1 1 loop are shown. Scores indicate $\log _{e}$ synthetic growth defects (red) or intergenic suppression (blue). A score of -4.6 indicates a 100× synthetic growth defect. Complete SGA results are presented in Supplementary Dataset 1. D, Gene Ontology (GO) Overrepresentation Test of the sly1 1 loop SGA dataset. Genes with $\log _{e}$ synthetic defect scores $\leq-0.5$ were included in the analysis. Bars show all GO-Slim Biological Process categories with statistically significant enrichment scores $\left({ }^{\star} p<0.05 ;{ }^{\star \star} p<10^{-2} ;{ }^{\star \star \star} p<10^{-6}\right)$. Pvalues were calculated using Fisher's exact test and adjusted for multiple comparisons (Bonferroni's correction; count $=732$ ). Additional details are presented in Supplementary Dataset 1. E SEC17 overproduction is toxic in cells expressing sly1 $\triangle l o o p$. sly $1 \Delta$ mutant cells were maintained with a counterselectable $S L Y 1$ balancer plasmid and transformed with single-copy plasmids bearing either 
SLY1 or sly1Dloop, as well as plasmids carrying SEC17 or sec17-FSMS (Schwartz and Merz, 2009). The balancer plasmid was ejected by plating dilutions on media containing 5-FOA and growth was assayed after 2 days of growth at $30^{\circ} \mathrm{C}$.

To assess the molecular mechanism of loss-of-function in Sly1 1 loop, we returned to the chemically defined fusion system. As shown in Fig.5, Sly1 1 loop elicited substantially slower fusion compared to wild-type Sly1. Moreover, Sly1 1 loop was unable to bypass the tethering requirement in vitro (Figs. 5A and C), consistent with its inability to suppress Ypt1 and Uso1 deficiencies in vivo. Importantly, in dose-response experiments both Sly $1 \Delta$ loop and wild-type Sly1 exhibited saturating fusion activity at $100 \mathrm{nM}$ (compare Sly1 $\Delta$ loop in Fig. 5 to wild type Sly1 in Fig. 3). Moreover, Sly1 $\Delta$ loop was properly folded as indicated by circular dichroism spectroscopy (Fig. 5E). Thus, the fusion defect of Sly1 $1 \Delta$ loop is not due to a major fraction of misfolded protein, and the sly1 1 loop allele is probably not a simple hypomorph. Instead Sly1 1 loop is, on a mole-per-mole basis, a less efficient stimulator of SNARE-mediated fusion
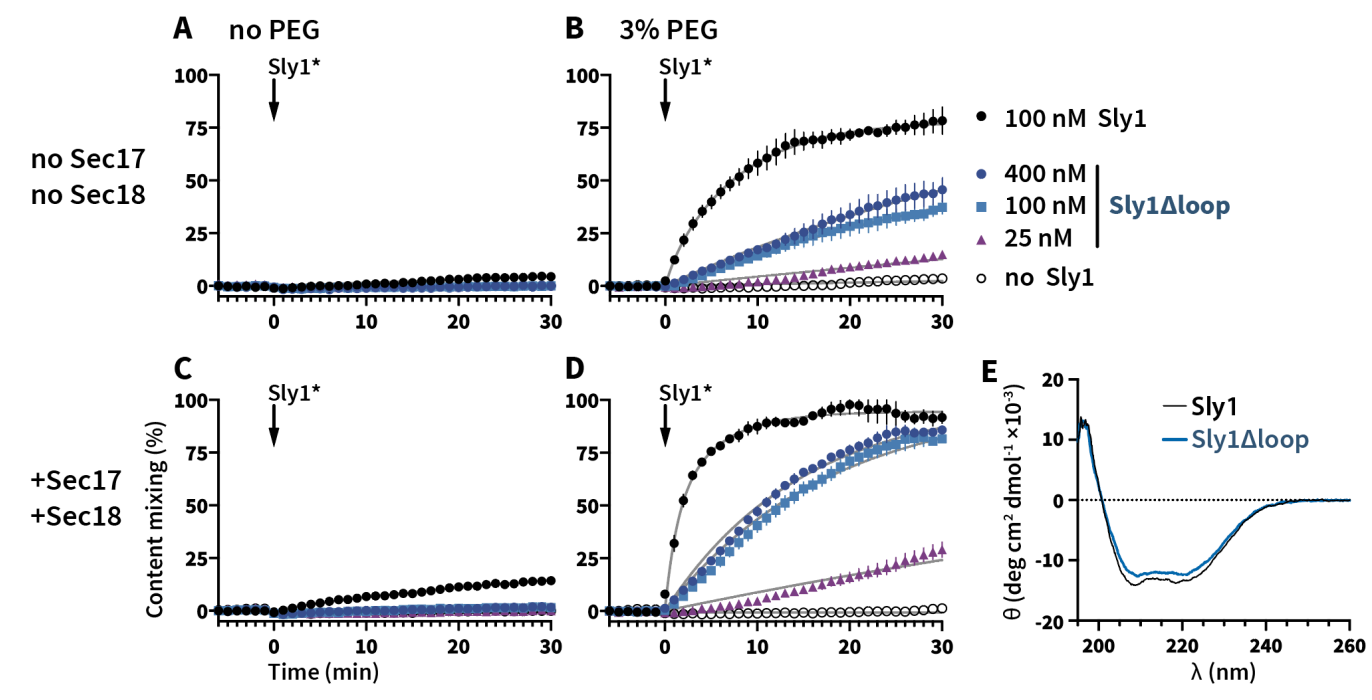

Fig. 5. The Sly1 regulatory loop has a positive function in vitro. A-D, fusion activity of Sly $1 \Delta$ loop versus wild type Sly1. Master mixes were assembled as in Fig. 3, and incubated for $5 \mathrm{~min}$ at $30^{\circ} \mathrm{C}$. Fusion was initiated by adding (arrows) Sly 1 or Sly $1 \Delta$ loop at the concentrations indicated in the legend adjacent to panel $\mathbf{B}$. Reactions were run in the absence $(\mathbf{A}, \mathbf{C})$ or presence $(\mathbf{B}, \mathbf{D})$ of $3 \%$ PEG; and in the absence $(\mathbf{A}, \mathbf{B})$ or presence $(\mathbf{C}, \mathbf{D})$ of Sec17, Sec18 (both $100 \mathrm{nM}$ ), and ATP. Points show mean \pm s.e.m. from three or more independent experiments; in some cases, the error bars are smaller than the symbols. Gray lines show least-squares nonlinear fits of a second-order kinetic model. E, Purified Sly $1 \Delta$ loop protein is folded. Circular dichroism spectra of wild type Sly1 and Sly1 $\Delta$ loop. The spectra are normalized to account for small differences in molecular mass and concentration. 
compared to the wild type. Taken together, the in vivo and in vitro data argue that the Sly1 regulatory loop is not only autoinhibitory, but that it also must harbor a positive fusionpromoting activity.

\section{The loop's positive function resides within ALPS-like helix $\alpha 21$}

The regulatory loop's most highly conserved region is helix $\alpha 21$ (Fig. 6A, B). Interestingly, none of the activating gain-of-function mutations isolated to date map to $\alpha 21$. On closer inspection we found that $\alpha 21$ is amphipathic (Fig. 6C). We therefore designed a mutant, Sly1pa21, in which helix $\alpha 21$ is mutated to render it polar rather than amphipathic. Unexpectedly, the sly1-pa21 allele caused recessive extremely slow growth or lethality (Fig. 6D) - a phenotype markedly more severe than that conferred by the sly $1 \Delta l o o p$ allele, which lacks the loop altogether.

Amphipathic helices operate as membrane recognition modules across a wide range of proteins, particularly within the early secretory pathway (Bigay and Antonny, 2012). This suggests a working model: the amphipathic $\alpha 21$ helix probes for the presence of an incoming vesicle and binds to the vesicle's membrane, pulling the loop away from the R-SNARE binding site. This disinhibits Sly1, allowing Sly1 to catalyze trans-SNARE complex assembly. In this model, the Sly1-pa21 protein is nonfunctional because $\alpha 21$ cannot recognize incoming vesicle membranes - and the loop is therefore locked into its auto-inhibited state. To test this hypothesis, we engineered a compound mutant, $S L Y 1-20-p \alpha 21$. This allele comprises both the activating Sly1-20 mutation (E532K) in $\alpha 20$, and the five $\alpha 21$-polar substitutions (Fig. 6d). Remarkably, SLY1-20-pa21 cells exhibited wild type growth (Fig. 6c). Unlike SLY1-20, however, SLY1-20-pa21 was unable to suppress the lethality of ypt1-3 or uso1A deficiencies (Supplementary Table 1), establishing that the amphipathic character of helix $\alpha 21$ is essential for Sly1-20 hyperactivity in vivo. 


\section{A} $\left.\right|_{\text {SLQNKSLLDGSDSAFKPSNLTLSGIYGLTEGKLQGG }} ^{\text {begin loop }} \frac{\alpha 21}{\text { disordered in crystal }} \frac{a 20}{\left.\right|_{\text {SLIKKLLPEKKTIPI }} ^{\text {end loop }}}$
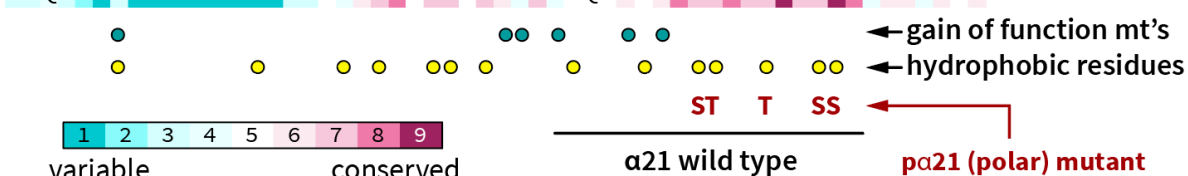

ST $T \quad$ SS

a21 wild type pa21 (polar) mutant

B

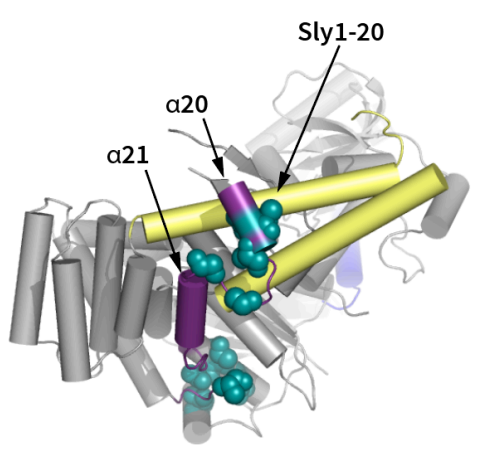

C

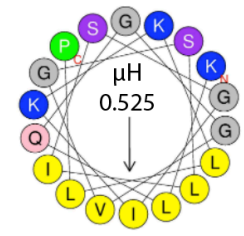

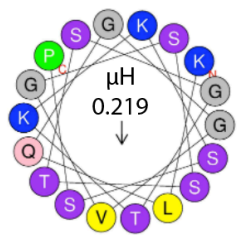

D sly1s

$S L Y 1$

$S L Y 1-20$

sly14loop

sly1-pa21

SLY1-20-pa21
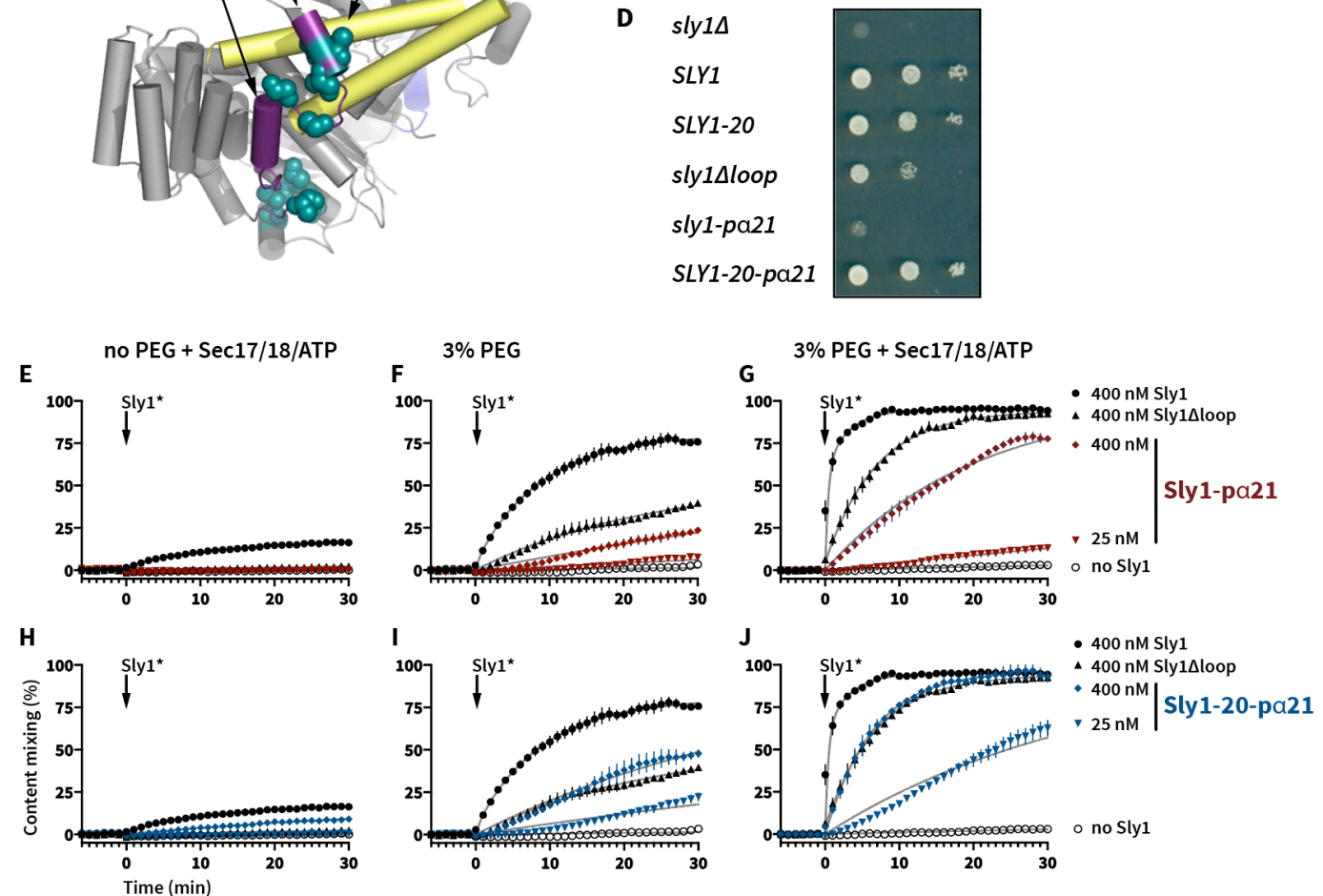

Fig. 6. Amphipathic helix $\mathbf{\alpha 2 1}$ is indispensable for normal Sly1 function. A, CONSURF analysis of evolutionary conservation within the Sly1 loop. Helix $\alpha 21$ is the most highly conserved portion of the loop. Locations of gain-of-function mutations, and hydrophobic residues within the loop, are indicated, as are the five substitutions in the Sly1-pa21 mutant. B, Position of helix $\alpha 21$ within Sly1. Note that no gain-of-function mutations within $\alpha 21$ have been identified. The loop is purple; the domain 3a templating domain is yellow. C, Helix $\alpha 21$ and residues immediately upstream have the potential to fold into a strongly amphipathic $\alpha$-helix. The helical wheel renderings comprise the region underlined in black and were produced using HELIQUEST; hydrophobic moment $(\mu \mathrm{H})$ is indicated. D, Growth phenotypes of cells carrying sly1-pa21, SLY1-20-pa21, and other alleles were assayed in a sly1 $\triangle$ strain with a $S L Y 1$ balancer plasmid, which is ejected in the presence of 5-FOA. (EJ)RPL fusion with (E-G) Sly1-pa and (H-J) the compound mutant Sly1-20-pa21. For reference, fusion is also plotted for Sly1 and Sly ${ }^{\Delta l o o p}$. Reactions were set up with (E,H) 0\% PEG, Sec17 and Sec18 (100 nM each), and ATP (1 mM); (F,I) 3\% PEG and no Sec17, Sec18 (100 nM each), or ATP; or (G,J F) 0\% 
PEG, Sec17 and Sec18 (100 nM each), and ATP (1 mM). Fusion was initiated at time $=0$ by adding Sly 1 or its mutants, at the concentrations indicated in the legends at the right side of the figure. Points show mean \pm s.e.m. from three or more independent experiments; in many cases the error bars are smaller than the symbols. Gray lines show least-squares nonlinear fits of a second-order kinetic model.

The in vivo results were closely mirrored in fusion experiments with RPLs (Fig. 6 E-G). Under every condition tested, Sly1-pa21 was less efficient at stimulating fusion than Sly1 $\Delta$ loop. Fusion in the presence of Sly1-pa21 was reduced in the absence or presence of PEG, as well as in the presence or absence of Sec17, Sec18, and ATP. In contrast to Sly1-pa21, the compound mutant Sly1-20-pa21 (Fig. 6 H-J) exhibited a greater ability to stimulate fusion under each of the tested conditions. Importantly, the behaviors of Sly1 $\Delta$ loop and the Sly1-20-pa21 compound mutant were similar. Hence, the amphipathic character of helix $\alpha 21$ is required for the loop's positive functions: activation and normal function of wild type Sly1, as well as hyperactivity of Sly1-20, both in vivo and in vitro.

To further test the hypothesis that the regulatory loop has a positive function we prepared chimeras, with fragments of the loop appended to the amino terminus of the Sly1 $\Delta$ loop mutant (Fig. 7A; Supplementary Table 3). In vivo, chimeras bearing the entire loop, or $\alpha 20-21$, or $\alpha 21$ alone, restored normal growth to Sly $1 \Delta$ loop (Fig. 7B). Mutation of five hydrophobic residues within $\alpha 21$ eliminated rescue by loop-SLY1Dloop or by $\alpha 20-21$ $S L Y 1 \Delta l o o p$. However, at $30^{\circ} \mathrm{C}$ the polar mutant pa21-SLY1Dloop grew almost as well as $\alpha 21$ SLY1Dloop. The mechanism of rescue by this mutant construct is unclear.

In vitro the $\alpha 20-21$-Sly1 $\Delta$ loop chimera drove almost wild-type fusion when added at $800 \mathrm{nM}$, whereas its polar mutant ( $\alpha 20-\mathrm{p} \alpha 21-\mathrm{Sly} 1 \Delta$ loop) phenocopied Sly1 $\Delta$ loop. Moreover, in contrast to the result obtained in vivo, a21-Sly1 $\Delta$ loop exhibited gain of function relative to Sly1 1 loop, while its polar mutant pa21-Sly $1 \Delta$ loop eliminated its gain-of-function relative to Sly1 $\Delta$ loop. Overall (with the interesting exception of the pa21-SLY1 $\Delta$ loop allele's in vivo phenotype), these results indicate that that the evolutionarily conserved portion of the Sly1 loop can partially replace the loop's positive function, even when appended to Sly1 at a nonnative location. 


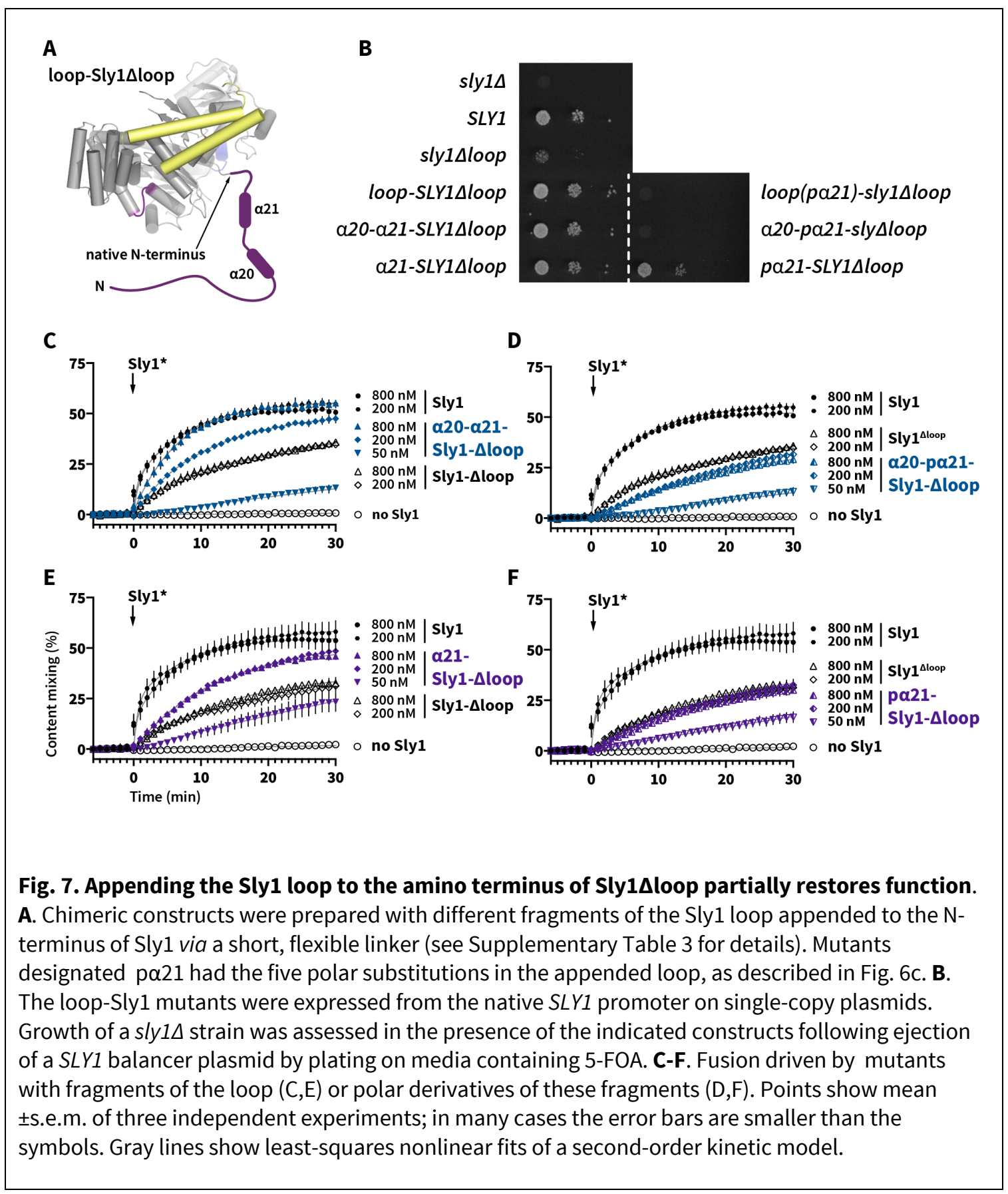




\section{Helix $\alpha 21$ can bind lipid bilayers directly, with preference for high curvature}

The above data suggest, but don't prove, that Sly1 helix $\alpha 21$ binds to membranes, and that the loop allows Sly 1 to tether vesicles. To test whether $\alpha 21$ binds to membranes we used a FRET assay. A peptide was synthesized comprising $\alpha 21$ and flanking residues, with an $\mathrm{N}$ terminal tetramethylrhodamine fluorophore (TMR- $\alpha 21)$. A control peptide, TMR-pa21, contained the same five substitutions as the Sly1-pa21 mutant (see Fig. 6A). Small unilamellar vesicles (SUVs) were prepared with $0.8 \%$ Texas Red-phosphatidylethanolamine (TRPE) to serve as a FRET acceptor for TMR. Representative emission spectra for the peptides and SUVs are shown in Fig. 8A. SUVs were prepared in two nominal diameters (30 and $200 \mathrm{~nm}$ ), and with either $6.7 \%$ or $30 \%$ ergosterol. When mixed with TRPE-doped SUVs, the $\alpha 21$-TMR peptide
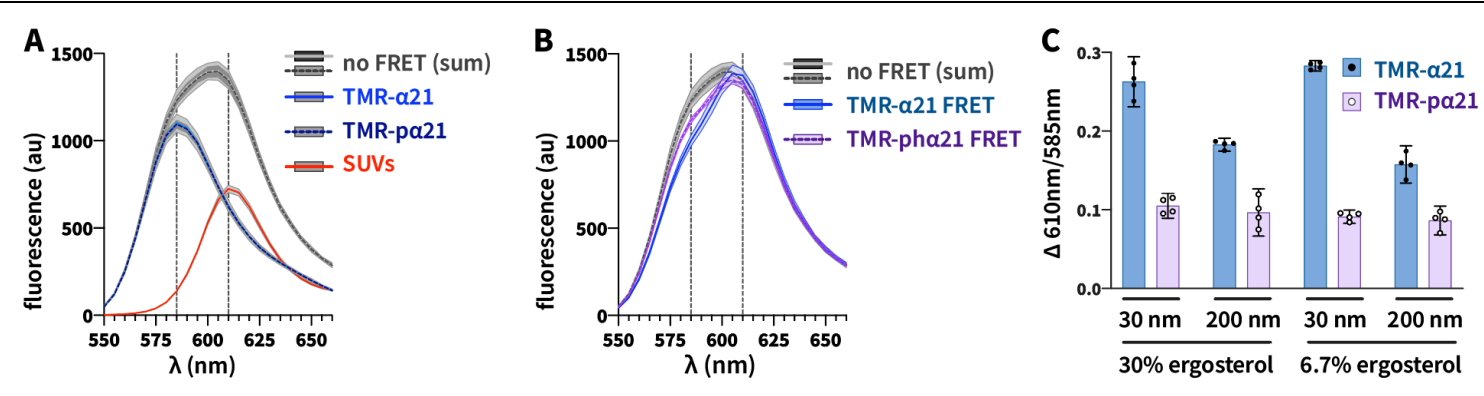

Fig. 8. Sly1 helix $\alpha 21$ binds membranes, with a preference for higher curvature. TMR- $\alpha 21$ and TMR-pa21 peptides were added to SUVs of nominal diameter 30 and $200 \mathrm{~nm}$, which contained 1\% TRPE as a fluorescence acceptor. A. Emission spectra of peptides or liposomes (30 nm diameter, $6.7 \%$ ergosterol) measured separately, and the sums of the peptide and liposome spectra. The sums represent the no-FRET condition. Both the TMR- $\alpha 21$ and TMR-pa21 spectra are plotted; they overlap almost exactly. Vertical dashed lines at $585 \mathrm{~nm}$ and $610 \mathrm{~nm}$ indicate emission peaks for labeled peptides and SUVs, respectively. B. Example of FRET data. Spectra from binding reactions containing SUVs (30 nm diameter, 6.7\% ergosterol, $500 \mu \mathrm{M}$ total lipid) and $25 \mu \mathrm{M}$ TMR- $\alpha 21$ or TMR-p $\alpha 21$ are shown. The no-FRET condition is shown for reference. C. Normalized FRET ratios for binding reactions containing the indicated combinations of SUVs and peptides, as in panel B. Traces and bars in $\mathrm{A}-\mathrm{C}$ show means and $\pm 95 \%$ confidence bands from 4 independent experiments.

generated a reproducible FRET signal, evident mainly as donor quenching (Fig. 8B,C). Under the same conditions, the control TMR-pa21 peptide exhibited smaller FRET signals. Moreover, the TMR- $\alpha 21$ peptide yielded a larger FRET signal with smaller SUVs, in both the $6.7 \%$ and $30 \%$ ergosterol conditions (Fig. 8C). In contrast, the TMR- pa21 FRET signals did not depend on the SUV diameter. We conclude that helix $\alpha 21$ binds directly to membranes through a mechanism involving the apolar residues within $\alpha 21$, and that it prefers to bind membranes with higher curvature. This is reminiscent of the behavior of ALPS domains, proposed to operate as 
membrane selectivity filters in the early secretory pathway. However, helix $\alpha 21$ and the Sly1 loop have a higher fraction of charged residues, and the apolar side chains are less bulky, than in canonical ALPS domains (Drin and Antonny, 2010). These differences may explain the apparent insensitivity of TMR- $\alpha 21$ binding to sterol concentration.

\section{Hyperactive Sly1* directly tethers vesicles to the Qa-SNARE}

Sly1 binds to the N-peptide-Habc domain of Sed5 (residues 1-210) with sub-nM affinity (Demircioglu et al., 2014; Grabowski and Gallwitz, 1997; Yamaguchi et al., 2002). Thus, we hypothesized that Sly1 may tether heterotypically, with one side of Sly1 binding to the Npeptide of Sed5 on the target membrane while the other side, via helix $\alpha 21$, binds directly to the membrane of the R-SNARE-bearing vesicle. To test this hypothesis we adapted a beadbased assay (Fig. 9A) previously used to study Rab-mediated tethering (Lo et al., 2012). First, GST-Sed5 cytoplasmic domain (GST-Sed5 cyt $_{\text {) }}$ or control GST protein were adsorbed to glutathione-agarose beads. Then Sly $1^{*}$ wild type or mutant proteins were added and allowed to bind to the immobilized GST-Sed $5_{\text {cyt. }}$. Finally, fluorescent vesicles or RPLs were added to the beads, incubated, and imaged by confocal microscopy. If Sly1 or its mutants mediate tethering between Sed5 and the membranes, the signal will be visible in confocal microscopy sections as an equatorial ring of fluorescence on the beads. Qualitative results with wild-type and mutant forms of Sly1 are shown in Fig. 9A. To quantify this tethering a bead spin-down assay was used (Fig. 9B-D). When Sly1-20, Sly1-T559I, or Sly1-D563G was added to the beads, robust tethering of SNARE-free small unilamellar vesicles (SUVS) was observed (Figs. 9A,B). Tethering was eliminated if either Sly1 or Sed5 ("GST control") was omitted. Tethering was dramatically attenuated with wild type Sly1, with Sly1 1 loop, or with Sly1-pa21. An intermediate tethering signal was observed with Sly1-20-pa21. The partial tethering observed with this compound mutant might be due to eight hydrophobic residues on the loop that are still present in the Sly1-pa21 and Sly1-20-pa21 mutants (see Fig. 6C). Robust tethering therefore requires that the loop be present, that the loop be open, and that helix $\alpha 21$ be amphipathic. Moeover, as in the peptide binding assays, Sly1-20 mediated tethering was most efficient with small-diameter vesicles, and was insensitive to sterol concentration (compare 
Figs. $8 \mathrm{C}$ and $9 \mathrm{C}$ ). Together these findings indicate that both helix $\alpha 21$ in isolation, and the Sly1 loop in the context of Sly1-20, sense membrane curvature.

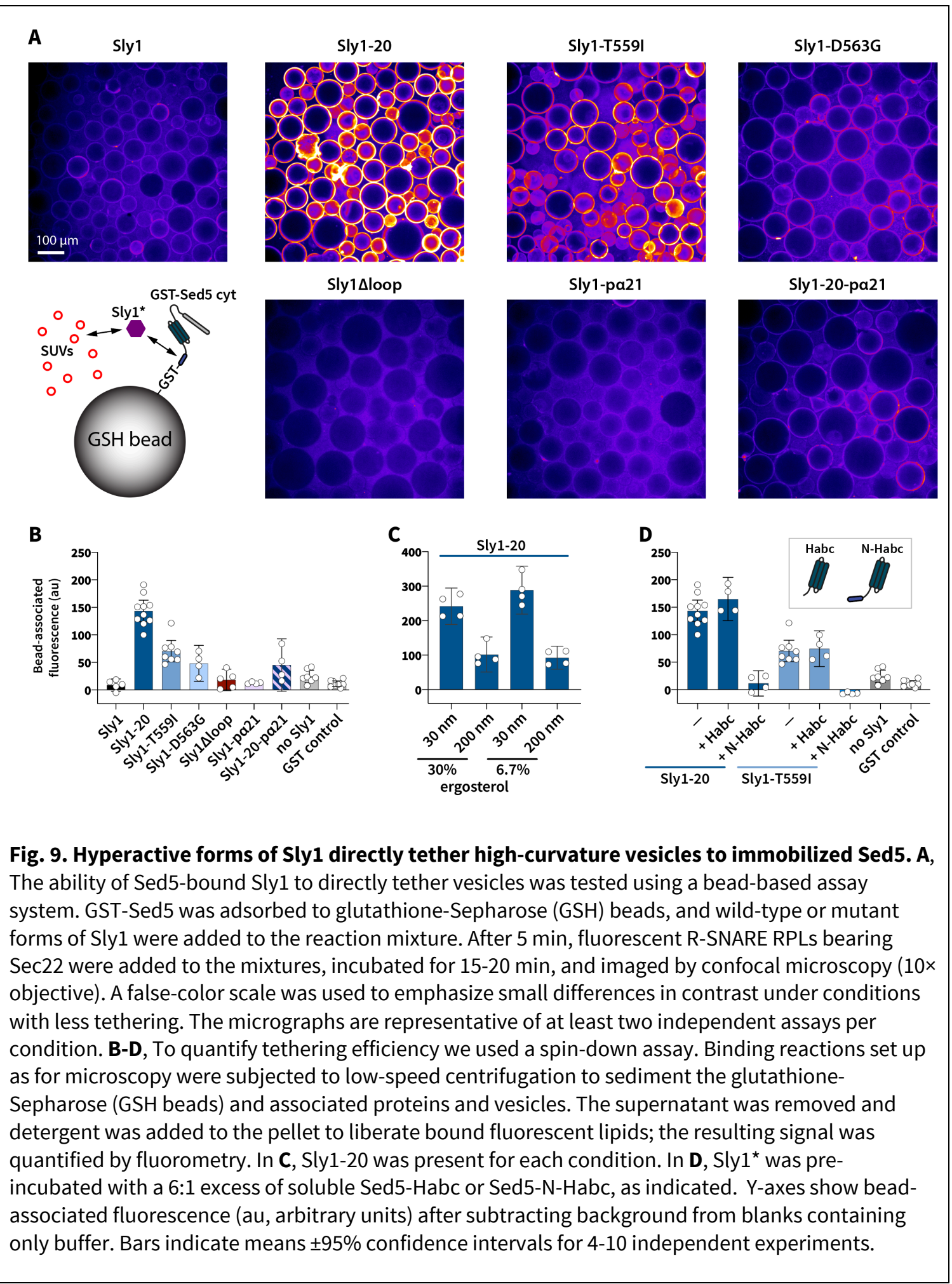


Weissenhorn, 2002; Demircioglu et al., 2014; Yamaguchi et al., 2002). To test the importance of this binding interaction in tethering, Sly1-20 was pre-incubated with a 6:1 molar excess of Sed5-N-Habc (aa 1-210; Fig. 9D). This abolished tethering. In contrast, tethering was not blocked by Sed5-Habc (aa 22-210), which lacks the N-peptide. Thus, to tether vesicles Sly1 must bind the N-peptide of the immobilized Qa-SNARE. Taken together the present and previously reported genetic data, and our assays of in vitro fusion, peptide binding, and tethering, all support the conclusion that the amphipathic helix $\alpha 21$ is necessary and sufficient for direct Sly1 binding to the incoming vesicle's lipid bilayer. This binding both derepresses Sly1 and allows it to tether incoming vesicles at close range.

\section{DISCUSSION}

$S L Y 1$ was identified through isolation of the $S L Y 1-20$ as a dominant single-copy suppressor of deficiency in YPT1, which encodes the yeast Rab1 ortholog. It soon became apparent that Ypt1 regulates ER-Golgi transport, and that $S L Y 1$ gain-of-function alleles might become hyperactive through the loss of negative regulation. The present experiments strongly support that hypothesis, but further demonstrate that helix $\alpha 21$ has at least two functions. First, $\alpha 21$ is needed for relief of Sly1 autoinhibition. Second, $\alpha 21$ has a positive function. Both $\alpha 21$ functions are essential for bypass of tethering requirements by $S L Y 1-20$, and both require the presence of conserved apolar residues within $\alpha 21$. The same apolar residues are required for direct binding of $\alpha 21$ to membranes. Sly1 mutants lacking the loop, or with a constitutively open loop that has reduced membrane affinity, exhibit loss of function relative to the wild type. Thus, the loop's ability to bind membranes has functions beyond relief of Sly1 autoinhibition. 
A Long-range tethering

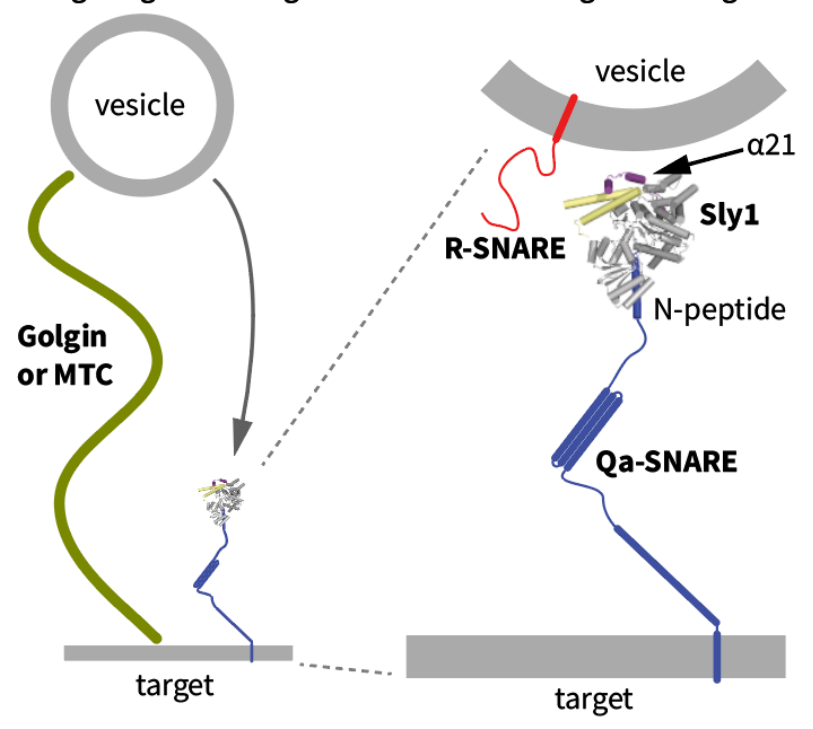

C
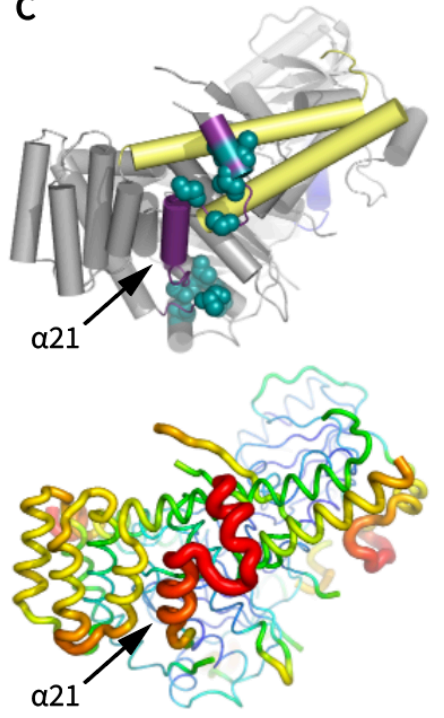

Fig. 10. Working model. A, Long-range tethering is mediated by coiled-coil Golgin family tethers and multi-subunit tethering complexes (MTC's). Flexibility or buckling of long-range tethers allows the vesicle to dwell in the region near Sly1 so that handoff can occur. B, Mechanism of close-range tethering. Sly1 is anchored to the N-terminal domain of the Qa-SNARE on the target membrane. Note that in the closed ground state, the loop and helix $\alpha 21$ sit opposite the N-peptide. Binding of $\alpha 21$ to an incoming vesicle's membrane pulls open the autoinhibitory loop and tethers the vesicle to Sly1, likely in a spatial orientation optimal for R-SNARE binding to Sly1 domain 3a (yellow). C. The Sly1 loop is conformationally heterogenous in the crystal structure (PDB 1MQS). The top rendering shows locations of single gain-of-function amino acid substitutions Sly1, as in Fig. 1B. The bottom rendering shows relative temperature (B) factors, encoded by color and backbone trace thickness. The poorly conserved amino terminal half of the loop was not resolved in the deposited structure.

In a working model (Fig. 10A), long-range tethers such as Uso1/p115 mediate initial capture of the vesicle, operating at ranges of 30-200 nm or more. Multisubunit Tethering Complexes (MTCs) including GARP, Dsl, and COG have long, floppy appendages (Chou et al., 2016; Ha et al., 2016; Ren et al., 2009). Golgins have an extended coiled-coil structure; their rod-like coiled-coil domains are interspersed with hinge-like domains. (Cheung and Pfeffer, 2016; Gillingham, 2018) In two cases, Golgin-210 and the Golgin-like endosomal tether EEA1, there is evidence that the hinges cause the tether to buckle or collapse, allowing the vesicle to approach the target membrane (Cheung et al., 2015; Murray et al., 2016). We propose that in the early secretory pathway long-range tethering factors hand vesicles off to Sly1, which would tether vesicles at a range of $\sim 15 \mathrm{~nm}$ from the target membrane to promote trans-SNARE complex assembly (Fig. 10B). The Sly1 loop's preference for small-diameter vesicles in our 
binding and tethering assays is reminiscent of the behavior of ALPS domains, which seem to operate as selectivity filters that recognize bulk physical properties of membranes in the early secretory pathway (Bigay et al., 2005; Bigay and Antonny, 2012; Drin et al., 2008; Magdeleine et al., 2016). We propose that Sly1's tethering function adds an additional layer of selectivity to this system.

In the anterograde ER-Golgi pathway both Sly1 and the Qa-SNARE Sed5 must be present on the Golgi acceptor membrane; they cannot fulfill their functions if located only on COPII-derived transport vesicles (Cao and Barlowe, 2000). Sly1 is anchored to Sed5 through a direct, sub-nanomolar interaction with the Sed5 N-terminal domain (Bracher and Weissenhorn, 2002; Demircioglu et al., 2014; Yamaguchi et al., 2002). As our experiments show, Sly1 binding to the Sed5 N-peptide is indispensible for Sly1-mediated tethering (Fig. 10B). However, a previous report argued that the Sed5-Sly1 interaction is of relatively minor importance (Peng and Gallwitz, 2004). In the companion manuscript (Duan et al., submitted) we show, consistent with tethering experiments presented here, that deletion of the Sed5 Npeptide severely impairs fusion in vitro and is lethal in vivo.

Sitting almost exactly opposite Sly1's N-peptide-binding cleft is the Sly1 loop (Fig. 10B). The loop is mobile: in the Sly1 crystal structure the poorly conserved $\mathrm{N}$-terminal half of the loop is unresolved, while the better-conserved C-terminal half of the loop is partially resolved but exhibits a high temperature (B) factor (Fig. 10C), an indication of conformational heterogeneity. We speculate that when Sly1 is in its auto-inhibited ground state, helix $\alpha 21$ undergoes a "logrolling" rotation about its long axis, intermittently exposing apolar side chains to probe for the presence of incoming vesicle membranes. Helix $\alpha 21$ binding to the vesicle bilayer has two consequences. First, the loop is pulled open, exposing the R-SNARE binding surface on Sly1. Second, the loop operates as a close-range tether, stabilizing the vesicle and target membrane within a distance sufficient to allow R-SNARE binding to Sly1 and the nucleation of a trans-SNARE complex on Sly1 domain 3a. The Sly1 loop might also constrain the rotational motion of Sly1 so that Sly1 is optimally oriented for productive R-SNARE binding. Although the available data are entirely consistent with this working model, we emphasize that many details are provisional and should be tested in future studies. 
In our in vitro tethering assays, Sly1-20 and other gain-of-function mutants allow efficient tethering, consistent with the ability of these mutants to suppress requirements for other tethering factors. In the same in vitro assays, however, wild type Sly1 tethers much less efficiently. This raises the question of whether close-range tethering is important for wild type Sly1. The Sly1 1 loop mutant cannot be auto-inhibited, yet it exhibits substantial tethering and fusion defects in vitro. In vivo, our SGA analyses revealed that the sly1 $\triangle l o o p$ allele exhibits synthetic sick or lethal interactions with dozens of genes involved in ER and Golgi traffic, including many genes that encode tethering factors or their regulators. In other words, when close-range tethering is prevented even partial defects in long-range tethering result in catastrophe and death. We suggest that a key function of Golgi long-range tethers is to allow incoming vesicles to dwell in the vicinity of Sly1 for long enough to allow inspection of vesicle membrane properties by $\alpha 21$, leading to loop opening, close-range tethering, R-SNARE binding, and assembly of a fusion-proficient trans-SNARE complex.

Additional mechanisms might contribute to the Sly1 loop's function. First, it is possible that as-yet unidentified proteins bind Sly1, contributing to loop opening and tethering. Second, when open (as in Sly1-20), the loop may be intrinsically disordered, generating a "steric cushion" that locally exerts force on the adjacent docked membranes (Busch et al., 2015; D'Agostino et al., 2017). Our evidence for such a steric cushion mechanism is equivocal. In vitro, the behavior of Sly $1 \Delta$ loop, which completely lacks the loop, and of Sly120-pa20, which has a full-length loop that is constitutively open but partially defective in membrane binding, exhibit similar defects in most assays. This would argue against the steric cushion hypothesis. In vivo, however, SLY1-20-pa20 allows almost wild type growth, while the sly1 1/loop mutant grows slowly. Finally, it is possible that $\alpha 21$ binding to the vesicle locally perturbs its membrane structure, lowering the energy barrier for the onset of lipid mixing. Additional work will be needed to evaluate these potential mechanisms.

Sly1 has been proposed to promote vesicle fusion in several ways. (i) The Golgi Qa/tSNARE Sed5 can adopt a tightly closed, autoinhibited conformation. Sly1 can open closed Sed5, allowing SNARE complexes to form more readily, at least in aqueous solution (Demircioglu et al., 2014; Kosodo et al., 1998). (ii) As we have shown here, helix $\alpha 21$ binding to membranes both de-represses and directly promotes Sly1 activity through a mechanism 
involving close-range vesicle tethering. (iii) Sly1 has conserved structural features that in Munc18-1 and Vps33 have been shown to catalyze trans-SNARE complex assembly through a Qa-R-SNARE templating mechanism. (iv) We have shown (again in aqueous solution but corroborated by genetic experiments) that Sly1 can decrease the rate of SNARE complex disassembly by Sec17 and Sec18. In the accompanying manuscript (Duan et al., submitted) we argue that that each of these mechanisms contributes to Sly1 function and that all are required for full Sly1 activity. In particular, we show that the close-range tethering mechanism characterized here is central Sly1's ability to selectively nucleate trans- versus cis-SNARE complexes.

The Sly1 loop is conserved among Sly1 homologs from yeast to human but is absent from representatives of the three other SM sub-families: Sec1/Munc18, Vps45, and Vps33. Why is the loop unique to Sly1? We suggest that Sly1 must function in a considerably broader variety of cellular and molecular contexts than other SM's. For example, the endosomal SM Vps45 associates with a scaffold protein, Vac1 (in mammals, Rabenosyn-5). Vac1 binds both Rab5 and phosphatidylinositol-3-phosphate, and might mediate close-range tethering in a manner analogous to the Sly1 loop (Burd et al., 1997; Peterson et al., 1999; Rahajeng et al., 2010; Tall et al., 1999). Similarly, Sec1 physically and functionally interacts with the exocyst tethering complex, and Vps33 is stably associated with Vps-C tethering complexes including HOPS and CORVET, which subsume both tethering and SNARE assembly functions (Morgera et al., 2012; Rieder and Emr, 1997). In the case of exocyst, the tethering activity is subject to autoinhibition, which is apparently released by engagement of rho family GTPases (Rossi et al., 2020).

In an interesting parallel, an ALPS-like domain within the HOPS subunit Vps41 was proposed to select high-curvature endocytic vesicles for docking and fusion (Cabrera et al., 2010). We also note that Munc18-1, despite lacking the Sly1-specific regulatory loop, is reported to tether vesicles in a reaction that requires at least the Qa-SNARE N-peptide and the R-SNARE on the opposing membrane (Arnold et al., 2017; Tareste et al., 2008). It is not clear whether Munc18-1 mediated tethering entails a direct interaction between Munc18-1 and the vesicle bilayer. This parallel may suggest that close-range tethering is a subreaction common to SM function. 
Which specific long-range tethers hand vesicles off to Sly1 for close-range tethering, docking, and fusion? Persuasive experiments show that Sly1 operates in concert with Ypt1 and Uso1 (yeast Rab1 and p115, respectively) on the anterograde ER-Golgi pathway (Cao and Barlowe, 2000). However, direct interactions between Sly1 and Ypt1 or Uso1 have not been detected. Binding interactions have been detected between human Sly1 and COG, and perhaps between yeast Sly1 and Dsl (Kraynack et al., 2005; Laufman et al., 2009). However, the positive suppressing interactions of $S L Y 1-20$, the negative synthetic sick or lethal interactions of sly1 1 loop, and the known SNARE interactions of Sly1, all point to Sly1 operating as "receiving agent" for vesicular traffic into the ER, early Golgi compartments, and perhaps later Golgi compartments as well. Additionally, several ER and Golgi tethers are reported to bind directly to Sly1 client SNAREs. Thus Sly1 might accept cargo containers presented by COG, GARP, TRAPP, Dsl, and the various Golgins. A significant challenge for the future is to identify the combinations of long-range tethers and SNAREs that operate in concert with Sly1, and to delineate the mechanisms that coordinate handoffs from long-range tethers to the core fusion machinery. 


\section{MATERIALS \& METHODS}

Yeast strains and SLY1 gain-of-function screen. We use standard Saccharomyces genetic nomenclature (Dunham et al., 2015). Dominant alleles, whether wild type or mutant, are named in uppercase type (e.g., SLY1-20); recessive alleles are named in lowercase (e.g., sly1 1 loop). Strains and plasmids used in this study are described in Supplementary Table 4. To obtain new suppressors of $u s O 1 \Delta$, a library of $S L Y 1^{*}$ mutant alleles was constructed using the GeneMorph II Random Mutagenesis Kit (Agilent \#200550). The SLY1 open reading frame was amplified using the "medium mutation rate" PCR protocol. Four mutagenic PCR pools were separately purified and cloned into a derivative of the yeast vector pRS415, which contained $431 \mathrm{bp}$ of the $S L Y 1$ promoter and $249 \mathrm{bp}$ of the $S L Y 1$ terminator, using traditional restriction-ligation methods. Aliquots of the pRS415::SLY1 mutant library ligation products were transformed into TOP10F' chemically competent $E$. coli cells, and 10 individual clones were Sanger sequenced to assess cloning fidelity and mutation frequency. Each clone sequenced contained the $S L Y 1$ open reading frame with 0-4 mutations, with about $50 \%$ of the clones containing mutations. After the $S L Y 1$ mutant library pools were verified, aliquots of the mutant library ligation products were transformed into Bioline Alpha-Select Gold Efficiency Competent $E$. coli cells. Transformant colonies were scraped from the LB + Amp transformation plates (maintaining four separate mutant pools), and allowed to grow for about two doublings. Plasmid DNA was extracted and purified from each of the pooled cultures using Qiaquick columns. 1ug of plasmid DNA from each $S L Y 1$ mutagenic pool was transformed into S. cerevisiae strain AMY2144 (CBY1297: uso1D pRS426::USO1). Transformant colonies were grown under selection for leucine auxotrophy, then replica plated to synthetic complete medium containing 5-FOA, and incubated for 2 days. Yeast colonies that grew on 5FOA (thus "kicking out" the WT copy of USO1) where struck out on -LEU plates, and plasmid DNA was purified from ten or more clones from each pooled library, using the Smash and Grab procedure. Plasmids were Sanger sequenced. On the basis of these results, pRS415::SLY1 single mutant alleles were constructed. Site-directed $S L Y 1^{*}$ mutants were constructed using PCR and Gibson assembly, and are described in Supplementary Tables 1-3. The second half of sly1-pa21 gene and its derivative SLY1-20-pa21 (see Supplementary Table 2) were ordered as a gBlock (IDT) and cloned into the BamHI and Ncol sites on the wild-type SLY1 plasmid. 
SGA analysis. A query strain (AMY2443) was constructed in the Y9205 genetic background (Tong and Boone, 2005), with sly14loop and a linked nourseothricin (NAT) marker integrated through allelic replacement at the native $S L Y 1$ locus. This query strain was crossed to the MAT a haploid deletion and DAmP libraries, where each individual genetic perturbation is marked with a KAN resistance marker (Breslow et al., 2008; Tong and Boone, 2005). Diploids were selected by robotic pinning (Singer RoToR) onto YPD + 100 mg/L clonNAT + 200 mg/L G418, then induced to sporulate by pinning to sporulation medium $(20 \mathrm{~g} / \mathrm{L}$ agar, $10 \mathrm{~g} / \mathrm{L}$ potassium acetate, $1 \mathrm{~g} / \mathrm{L}$ yeast extract, $0.5 \mathrm{~g} / \mathrm{L}$ glucose, $0.1 \mathrm{~g} / \mathrm{L}$ amino acid supplement [ $2 \mathrm{~g}$ histidine, $10 \mathrm{~g}$ leucine, $2 \mathrm{~g}$ lysine, $2 \mathrm{~g}$ uracil]) and growth at room temperature for 5 days. Spores were subsequently pinned to haploid selection medium (SD -His/Arg/Lys $+50 \mathrm{mg} / \mathrm{L}$ canavanine +50 $\mathrm{mg} / \mathrm{L}$ thialysine) and MAT a meiotic progeny grown for 2 days at $25^{\circ} \mathrm{C}$. This haploid selection step was repeated, and the resulting colonies imaged using a Phenobooth (Singer) imaging system. These colonies encompass all potential meiotic progeny and serve as the control strains for phenotypic normalization. Haploid double mutants carrying both the KAN deletion allele and the sly1 $\triangle l o o p:: N A T$ allele were selected by pinning meiotic progeny to double selection medium (SD/MSG -His/Arg/Lys $+50 \mathrm{mg} / \mathrm{L}$ canavanine $+50 \mathrm{mg} / \mathrm{L}$ thialysine $+100 \mathrm{mg} / \mathrm{L}$ clonNAT $+200 \mathrm{mg} / \mathrm{L} \mathrm{G418).} \mathrm{After} 2$ days of growth at $25^{\circ} \mathrm{C}$, this selection step was repeated and duplicate plates incubated at either $30^{\circ} \mathrm{C}$ or $37^{\circ} \mathrm{C}$. Plates were imaged using the Phenobooth system, and colony size differences calculated using PhenoSuite software and web app (https://singerinstruments.shinyapps.io/phenobooth/).

Protein purification. Full-length SNARE proteins were produced as previously described (Furukawa and Mima, 2014) with modifications. E. coli Rosetta2 (DE3) pLysS cells (Novagen) harboring each of the SNARE expression plasmids with $3 \mathrm{C}$ protease-cleavable $\mathrm{N}$-terminal tags (pET-41/GST-His 6 for SEC22 and pET-30/His 6 for SED5, BOS1 and BET1) were inoculated from a 1:1000 dilution of the starter culture grown in MDAG-135 medium (Studier, 2005) into $1 \mathrm{~L}$ of Terrific Broth supplemented with $100 \mu \mathrm{g} / \mathrm{mL}$ Kanamycin and $34 \mu \mathrm{g} / \mathrm{mL}$ Chloramphenicol and grown at $37^{\circ} \mathrm{C}, 275 \mathrm{rpm}$ until OD600 reached $\sim 1$. Cultures were then induced with $1 \mathrm{mM} \mathrm{IPTG}$ for $3 \mathrm{~h}$ at $37^{\circ} \mathrm{C}$. Cultures were harvested at $5000 \times \mathrm{g}$ and cell pellets were snap frozen with liquid nitrogen. Each liter yielded $\sim 10 \mathrm{~g}$ of wet cells, which were stored at $-70^{\circ} \mathrm{C}$. For 
purification, the frozen pellets were warmed to $-10^{\circ} \mathrm{C}$ and broken up into small pieces with a metal spatula, then resuspended at a ratio of $5 \mathrm{~mL}$ of buffer per $\mathrm{g}$ of cell paste in $1 \times$ SNARE buffer $\left(20 \mathrm{mM} \mathrm{Na} \mathrm{PO}_{4}, 500 \mathrm{mM} \mathrm{NaCl}, 10 \%\right.$ (m/v) glycerol, $1 \mathrm{mM} \mathrm{DTT}, \mathrm{pH}$ 7) supplemented with 30-40 mM imidazole, $0.25 \mathrm{mg} / \mathrm{mL}$ chicken egg lysozyme, $125 \mathrm{U}$ benzonase per $\mathrm{g}$ of cells, and $1 \times$ Sigmafast Protease inhibitor cocktail. $4 \mathrm{~mL}$ (1/10 volume) of $1 \mathrm{M} \mathrm{n}$-octyl- $\beta$-D-glucopyranoside in $\mathrm{H}_{2} \mathrm{O}$ ( $\beta$-OG, Anatrace) was added to $100 \mathrm{mM}$ final concentration; the suspension was rotated at room temperature for $25 \mathrm{~min}$ to allow detergent-aided enzymatic lysis. Lysates were clarified at $16,500 \times \mathrm{g}, 4^{\circ} \mathrm{C}$ for $10 \mathrm{~min}$, transferred to clean centrifuge tubes and centrifuged again for $20 \mathrm{~min}$. Clarified lysates were batch-bound with $2 \mathrm{ml}$ of $\mathrm{Ni}$-Sepharose HP equilibrated in $1 \times$ SNARE buffer with $\beta$-OG for 30 minutes. SNARE-bound resin was washed in plastic disposable columns with $25 \mathrm{~mL}$ of SNARE buffer supplemented with $\beta-O G$ and 60-100 mM imidazole. SNARE proteins were eluted with SNARE buffer supplemented with $\beta-O G$ and 200$300 \mathrm{mM}$ imidazole, and snap frozen in liquid nitrogen. Purified protein was quantified using by absorbance at $280 \mathrm{~nm}$ and purity was assessed with SDS-PAGE with Coomassie blue staining. Protein aliquots were stored at $-70^{\circ} \mathrm{C}$ until reconstitution. We note that $3 \mathrm{C}$ protease caused substantial unintended cleavage of Bos 1 in its N-terminal linker domain due to a cryptic $3 \mathrm{C}$ site (148-GLPLYQ/GL-155). Mutation of the poorly-conserved residue Q153 to aspartic acid eliminated unintended proteolysis.

Soluble domains of Sed5 were expressed from the pET-30 vector (for $\mathrm{H}_{6}-\mathrm{Habc}$ and $\mathrm{H}_{6}{ }^{-}$

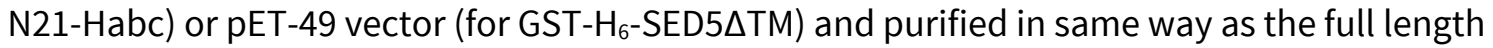
protein except that the temperature was lowered to $35^{\circ} \mathrm{C}$ prior to induction, the buffers did not contain $\beta-O G$, and lysis was performed using Emulsiflex- $\mathrm{C} 5$ high pressure homogenizer (Avestin). Eluted protein was exchanged into FB160M1 (20 mM HEPES·KOH, $160 \mathrm{mM} \mathrm{KOAc}$, $10 \%$ (m/v) Glycerol, 1mM MgOAc 2 , pH 7) using a PD-10 desalting column. Precipitated material was removed by centrifugation at $10,000 \times \mathrm{g}$ for 10 minutes and soluble protein aliquots were snap frozen in liquid nitrogen in $250 \mathrm{ul} \mathrm{PCR}$ tubes and stored at $-80^{\circ} \mathrm{C}$. Sec22(SNARE)-GFP-His 8 was expressed from the PST50Trc1 vector in Rosetta2(DE3) cells grown in ZYM-5052 autoinduction media (Studier, 2005) supplemented with carbenicillin $(100 \mu \mathrm{g} / \mathrm{mL}$ ) and chloramphenicol $(34 \mu \mathrm{g} / \mathrm{mL})$ overnight $(>16 \mathrm{~h})$ at $30^{\circ} \mathrm{C}$ from a $1: 1000$ dilution of starter culture. Cells were harvested and protein was purified as for soluble domains of Sed5. Sec17 was 
purified as described (Schwartz and Merz, 2009) except that the culture was grown in ZYM5052 autoinduction media (Studier, 2005) at $37^{\circ} \mathrm{C}$ until $\mathrm{OD}_{600 \mathrm{~nm}}$ was $\sim 0.8$; temperature was then lowered to $18^{\circ} \mathrm{C}$ and the culture was incubated for $\sim 24$ hours. Sec18 was purified as described (Haas and Wickner, 1996).

Sly1 and its mutants were expressed in Rosetta2(DE3) cells from pHIS-Parallel1 vectors (Lobingier et al., 2014; Sheffield et al., 1999). Frozen glycerol stocks were used to inoculate overnight starter cultures at $37^{\circ} \mathrm{C}$ in MDAG-135 containing $100 \mathrm{mg} / \mathrm{L}$ carbenicillin and $50 \mathrm{mg} / \mathrm{L}$ chloramphenicol (Studier, 2005). Each starter culture was diluted 1/1000 to seed 1-2 L of Terrific Broth containing $100 \mathrm{mg} / \mathrm{L}$ carbenicillin and $34 \mathrm{mg} / \mathrm{L}$ chloramphenicol. These cultures were grown in an orbital shaker $\left(37^{\circ} \mathrm{C}, 275 \mathrm{rpm}\right)$ to $\mathrm{OD}_{600 \mathrm{~nm}} \sim 1$. Cultures were then transferred to a prechilled shaker at $16^{\circ} \mathrm{C}$ for $1 \mathrm{~h}$ before induction with $0.1-1 \mathrm{mM} \mathrm{IPTG}$ for $18 \mathrm{~h}$. Cells were sedimented and resuspended in cold Sly1 buffer $\left(20 \mathrm{mM} \mathrm{Na} \cdot \mathrm{PO}_{4}, 500 \mathrm{mM} \mathrm{NaCl}, 10 \%\right.$ (m/v) glycerol, $1 \mathrm{mM} \mathrm{DTT}$, pH 7) supplemented with $30 \mathrm{mM}$ imidazole, $0.25 \mathrm{mg} / \mathrm{mL}$ chicken egg lysozyme and $1 \times$ Sigmafast Protease inhibitor cocktail at a ratio of $5 \mathrm{~mL}$ of buffer per $\mathrm{g}$ of cell paste. The cells were lysed by passing through Emulsiflex-C5 high pressure homogenizer (Avestin) 2-4 times and the lysate was clarified by centrifugation at $\left(16,500 \times \mathrm{g}, 25 \mathrm{~min}, 4^{\circ} \mathrm{C}\right)$. Clarified lysate from $1 \mathrm{~L}$ of culture ( $2 \mathrm{~L}$ for Sly $1 \Delta$ loop and Sly1-20) was bound in batch with 1 $\mathrm{mL}$ equilibrated $\mathrm{Ni}^{2+}$-Sepharose HP resin (GE) for $30 \mathrm{~min}$ at $4^{\circ} \mathrm{C}$. Sly1-bound resin was collected in a $25 \mathrm{~mL}$ disposable Econo-Pac column (Bio-Rad) by gravity and washed with 25 $\mathrm{mL}$ of SLY1 buffer supplemented with $50 \mathrm{mM}$ imidazole at pH 7. Sly1 was eluted with Sly1 buffer supplemented with $300 \mathrm{mM}$ imidazole pH 7 in $0.5 \mathrm{~mL}$ fractions. Most of the protein eluted in fractions 3-7. Sly1 was exchanged into FB160M1 (20 mM HEPES·KOH, $160 \mathrm{mM} \mathrm{KOAC,}$ 10\% m/v Glycerol, 1 mM MgOAc 2 , pH 7) using a PD-10 column (GE Healthcare). Precipitated material was removed by centrifugation at $10,000 \times \mathrm{g}$ for 10 minutes and soluble protein were diluted or concentrated to $\sim 2.4 \mathrm{mg} / \mathrm{mL}$. Aliquots were snap-frozen in liquid nitrogen in thinwall PCR tubes and stored at $-70^{\circ} \mathrm{C}$.

Recombinant HRV3C protease was prepared either as an N-terminal His8-tag fusion (AMP2019) or an N-terminal GST-His $6_{-}$(Thrombin) fusion (AMP2016). $1 \mathrm{~L}$ of 1/1000 dilution of an overnight culture of Rosetta2(DE3) cells harboring the expression plasmid was grown overnight at $37^{\circ} \mathrm{C}$ in ZYM-5052 autoinduction media with $100 \mu \mathrm{g} / \mathrm{mL}$ kanamycin and $34 \mu \mathrm{g} / \mathrm{mL}$ 
chloramphenicol. Cells were centrifuged, resuspended in 4 times the volume of Lysis buffer (50 mM Tris- $\mathrm{HCl}$ pH 8.0, $300 \mathrm{mM} \mathrm{NaCl}, 10 \%$ glycerol, $1 \mathrm{mM} \mathrm{DTT}$, and no protease inhibitors) supplemented with $15 \mathrm{mM}$ imidazole and $0.5 \mathrm{mg} / \mathrm{mL}$ lysozyme, and lysed using Emulsiflex-C5 high pressure homogenizer (Avestin). Clarified lysate was incubated with $3 \mathrm{~mL} \mathrm{Ni}^{2+}$-Sepharose HP (GE) for 30 min and strained in a disposable column. Resin was washed thoroughly with Lysis buffer supplemented with 40-60 mM imidazole and protein was eluted with $200 \mathrm{mM}$ imidazole in about $7.5 \mathrm{~mL}$. Concentrated fractions were combined and EDTA was added to 1 $\mathrm{mM}$. The yield was $\sim 100 \mathrm{mg}$ of purified protease per $1 \mathrm{~L}$ of culture. Purified protease was diluted to $10 \mathrm{mg} / \mathrm{ml}$ and exchanged into storage buffer (50 mM Tris- $\mathrm{HCl}(\mathrm{pH} 8.0), 150 \mathrm{mM} \mathrm{NaCl}$, $1 \mathrm{mM}$ EDTA, $1 \mathrm{mM}$ DTT, and $20 \%$ glycerol.), frozen in liquid $\mathrm{N}_{2}$, and stored at $-80^{\circ} \mathrm{C}$. Protease activity of the preparations was assayed using a homemade assay based on a linked FRET pair of fluorescent proteins (Evers et al., 2006), modified with an HRV 3C-cleavable linker. Reduction in FRET due to proteolysis was monitored in real time using a SpectraMax Gemini microplate reader (Molecular Devices).

GST-His 6 was expressed and purified using conventional $\mathrm{Ni}^{2+}$ IMAC chromatography methods. Protein was exchanged into FB160M1 before freezing in liquid $\mathrm{N}_{2}$ and stored at $-80^{\circ}$ C.

Circular Dichroism Spectroscopy. Purified SLY1wt or SLY1 1 loop was exchanged into CD buffer (20 mM Na-Pi, $100 \mathrm{mM} \mathrm{NaCl}, \mathrm{pH} 7.2$ ), diluted to $0.2 \mathrm{mg} / \mathrm{mL}$, and loaded into a $0.1 \mathrm{~cm}$ path length cuvette. Spectroscopy was performed using a J-1500 CD Spectrophotometer (JASCO) at $25^{\circ} \mathrm{C} . \mathrm{CD}$ and absorbance were measured from $\lambda=195$ to $260 \mathrm{~nm}$ in steps of $0.1 \mathrm{~nm}$. The protein concentration during each read was determined from absorbance at $205 \mathrm{~nm}$, using the extinction coefficient at $205 \mathrm{~nm}$ calculated by the Anthis and Clore method (http://nickanthis.com/tools/a205.html) for each protein. Molar ellipticity for each protein was calculated by dividing the $C D$ at each wavelength by the cuvette pathlength and protein concentration. Mean residue ellipticity for each protein was calculated by dividing the Molar ellipticity by the number of amino acids per protein.

Preparation of RPLs. The FB160 buffer system and lipid mixtures used here are derived from B88 buffer, used extensively in COPII vesicle budding assays (Baker et al., 1988), and from lipidomic studies. The ER lipid mix is based on "Major-Minor" mixtures used for COPII budding 
(Antonny et al., 2001; Matsuoka et al., 1998), while the Golgi-mix is based on lipidomic surveys (Klemm et al., 2009; Schneiter et al., 1999). In particular, the study of Schneiter et al. used a highly enriched Golgi fraction known to be competent for docking and fusion of COPII carrier vesicles (Lupashin et al., 1996). Relatively high concentrations of ergosterol were used based prior work on COPII budding, which demonstrated that higher sterol levels yielded more morphologically homogenous COPII budding profiles (Matsuoka et al., 1998). In pilot studies, however, RPLs prepared with lower ergosterol concentrations exhibited similar fusion characteristics, including Sly1 and PEG dependence, as the high-sterol RPLs used in the experiments presented here. Lipids were obtained from Avanti Polar Lipids as chloroform stocks (Alabaster, AL) except for ergosterol, which was from Sigma-Aldrich. Supplementary Table S5 lists the proportions, working stocks, and volumes of lipids and detergent used to prepare ER-mix and Golgi-mix RPLs. Lipid stocks were prepared or purchased in chloroform, except for ergosterol and phosphatidylinositol-4-phosphate, which were dissolved in 1:1 chloroform:methanol. $\beta$-OG stock solutions were prepared in methanol. Lipid-detergent films were prepared by transferring lipid and $\beta$-OG stocks to a glass vial (typically, $8 \mu \mathrm{mol}$ total lipids and $70 \mu \mathrm{mol} \beta-\mathrm{OG})$. The mixture was dried under a nitrogen stream; residual solvent was removed using a Speedvac ${ }^{\mathrm{TM}}$ evaporator. The lipid-detergent film was hydrated and solubilized with $400 \mu \mathrm{L} 5 \times$ FB160M1 by three cycles of bath sonication and shaking. To the lipid$\beta$-OG mixture, content mixing FRET reporters were then added $(500 \mu \mathrm{L}$ of $4 \mathrm{mg} / \mathrm{mL}$ solution of R-phycoerythrin-biotin conjugate, or $296 \mu \mathrm{L}$ of $2 \mathrm{mg} / \mathrm{mL}$ Alexa-Streptavidin; both reagents from Thermo/Molecular Probes). SNARE stocks in SNARE elution buffer with $\beta$-OG were then added to a final molar ratio of 1:600 (each Q-SNARE) or 1:300 (Sec22) to total phospholipids. Water was used to fill the headspace necessary to dilute $5 \times$ FB160M1 buffer to $1 \times$ concentration $(2 \mathrm{~mL}$ final volume). Mixtures were nutated for 30 minutes before recombinant $3 \mathrm{C}$ protease was added (in 1:10 ratio to total SNAREs) to cleave affinity tags from the SNARE proteins during dialysis. The resulting mixtures were dialyzed (20 kDa cutoff) for $\sim 18 \mathrm{~h}$ at $4^{\circ} \mathrm{C}$ in the dark against 250 volumes of FB160M1 containing $2 \mathrm{~g}$ BioBeads SM2 (Bio-Rad, Hercules, CA) per $2 \mathrm{~mL}$ of RPL mixture. The RPL mixture was then separated from unencapsulated content mixing probe by floating the RPLs up a step gradient of iso-osmotic Histodenz (35/25/0\%) in FB160M1 (SW60Ti rotor at 55k rpm for $90 \mathrm{~min}$ ), harvested, and diluted to $2 \mathrm{mM}$ phospholipid. Phospholipid was quantified by measuring the fluorescence of the membrane fluorophore, initially verified by 
inorganic phosphate analysis (Chen et al., 1956). $32 \mu \mathrm{L}$ aliquots of RPLs were transferred to thinwall PCR tubes and frozen by immersion in liquid $\mathrm{N}_{2}$. RPLs prepared by this method and stored at $-80^{\circ} \mathrm{C}$ were stable and fusion-competent, with minimal leakage of encapsulated FRET probes, for at least one year.

RPL fusion assays. Unless noted otherwise, a standard order-of-addition was always used to initiate RPL assays. $250 \mu \mathrm{M}$ (final phospholipid) of each RPL was premixed with PEG6K and other fusion components such as Sec17, Sec18 and ATP. Fusion assays were performed in 20 $\mu \mathrm{L}$ sample volumes in 384-well plates (Corning \#4514). The reactions were monitored in a plate-based fluorimeter (Molecular Devices Gemini XPS or EM) for 5 min to establish a baseline, then Sly1 was added to initiate fusion. Except as noted, the moment of Sly1 addition was defined as time $=0$. Lipid mixing was monitored with $\mathrm{Ex}_{370 \mathrm{~nm}}$ and $\mathrm{Em}_{465 \mathrm{~nm}}$. Content mixing was monitored with $\mathrm{Ex}_{565 \mathrm{~nm}}$ and $\mathrm{Em}_{670 \mathrm{~nm}}$. Graphs show mean \pm s.e.m. of $\mathrm{n} \geq 3$ independent assays. Curves on the graphs show a second-order kinetic model fit to each dataset using a weighted least-squares algorithm in GraphPad Prism. Some experiments were run in both the presence and absence of unlabeled streptavidin, to assess leakage of RPL aqueous contents. For content mixing, typical signal for a complete reaction over background (e.g., no Sly1) exceeded 50:1.

Yeast growth assays. Yeast strains containing pRS426::USO1 or pRS416::YPT1 and pRS415::SLY1 mutant plasmids were grown in -LEU liquid media, then diluted using a 48-pin manifold or a multichannel pipettor onto 5-FOA plates. The 5-FOA plates were grown at restrictive or non-restrictive temperatures, as indicated. Growth was scored relative to positive and negative control strains after 2-3 days.

Peptide-liposome binding assay. To prepare small Texas RED-DHPE labeled unilamellar vesicles, lipid chloroform stocks were mixed using Hamilton syringes in glass vials, dried under a nitrogen stream, and residual solvent was removed in a Speedvac ${ }^{\mathrm{TM}}$ concentrator. The resulting lipid films were rehydrated with FB160M1 and either sonicated or extruded using an Avanti mini extruder with $0.03,0.05$ or $0.2 \mu \mathrm{m}$ polycarbonate filters (Whatman). Peptides were custom-synthesized with a tetramethylrhodamine (TMR) fluorophore at the N-terminus, and were $>98 \%$ pure by HPLC. The fluorophore is zwitterionic and does not change the net charge 
$(+1)$ of the peptides. Emission spectra were acquired using a Molecular Devices Gemini XPS fluorescence spectrometer. FRET ratios were calculated as the ratio of fluorescence emission at 610 and $585 \mathrm{~nm}$. The data were normalized by comparing each sample to the corresponding no-FRET condition (sum of the FRET signals for each peptide and SUV, acquired separately):

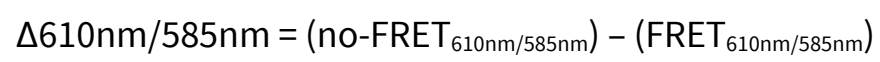

Bead-based tethering assays. Beads were prepared in $100 \mu \mathrm{L}$ (10 reactions) or $1 \mathrm{~mL}(100$ reactions) batches. In small disposable spin columns, $100 \mu \mathrm{L}$ of beads were washed in FB160M1 supplemented with 1\% (m/v) bovine serum albumin (FB160M1BSA), and were loaded with $100 \mu \mathrm{g}$ of GST-Sed5 cytoplasmic domain (1/5 of the resin's nominal binding capacity; 150 pmol protein per $10 \mu \mathrm{L}$ resin in a $1 \times$ reaction), in a volume of $500 \mu \mathrm{L}$ FB160BSA; this mixture was incubated, with slow agitation, for $30 \mathrm{~min}$ at room temperature. Unbound material was removed by gentle centrifugation ( $70 \times \mathrm{g}, 10$ seconds), the beads were washed once with FB160BSA, and the beads were then blocked by adding excess recombinant GST$\mathrm{His}_{6}$ protein (1.25 mg; $2.5 \times$ the resin's nominal binding capacity) in FB160BSA, in 500ul final volume. Unbound GST-H6 was not removed. The bead-SED5-GST suspension was stored at $4^{\circ}$ for up to a week. For tethering assays, $1 \times$ reaction aliquots of the bead-SED5-GST suspension (50 $\mu \mathrm{L}$, containing $\sim 10 \mu \mathrm{L}$ packed beads) were transferred to $250 \mu \mathrm{L}$ PCR tubes, then Sly1* (75 pmol; a 1:2 molar ratio to Sed5 ${ }_{\text {cyt }}$ ) was added to each reaction tube in $50 \mu \mathrm{L}$ volume, allowing the Sly $1^{\star}$ to bind to the immobilized GST-Sed $5_{\text {cyt }}$ in $100 \mu \mathrm{L}$ final volume. For competition experiments the Sly $1^{*}$ was pre-incubated with a 6 -fold molar excess ( $450 \mathrm{pmol}$ ) of Sed5 Habc or $\mathrm{N}$-Habc domain for 10 min at room temperature before adding the Sly $1^{*}$-competitor mixture to the beads. Tethering was initiated by adding Texas-red-DHPE labeled SUVs to each $1 \times$ tethering reaction (1-6 $\mu \mathrm{L}$ depending on stock concentration). The tethering reactions were incubated for 15-20 min at room temperature, then transferred to wells of chambered coverslips that had been pre-incubated with FB160BSA for at least $20 \mathrm{~min}$. These preparations were observed at ambient temperature $\left(23 \pm 2^{\circ} \mathrm{C}\right)$ using a Nikon Ti2 microscope equipped with a Yokogawa CSU-X1 spinning disk confocal unit, a Toptica iChrome MLE laser combiner and launch; 405, 488, 561, and 647 nm diode lasers (Coherent); a Finger Lakes high-speed emission filter wheel; and a Mad City piezoelectric Z-stage. The microscope was controlled by Nikon 
Elements software and data analysis and figure preparation was done with the Fiji package of Image/J software and plug-ins. Tethering reactions were observed using a 10×0.30 NA Plan Fluor objective and an Andor 888 EMCCD camera operated at an EM gain of 300 with $200 \mathrm{~ms}$ exposure per frame.

Tethering was also quantified using bead spin-down assays. Binding reactions were initiated as in the microscopy-based tethering experiments. To quantify SUVs tethered to the beads, the beads were washed once in $1.3 \mathrm{~mL}$ of FB160BSA and then sedimented for $1 \mathrm{~min}$ at $500 \times \mathrm{g}$ in a swinging-bucket rotor. The supernatant was carefully removed, and resin-bound lipids were eluted from the beads with $50 \mu \mathrm{L}$ of BugBuster protein extraction reagent (Millipore). The beads were again sedimented. To quantify the amount of eluted TRPE lipid, $20 \mu \mathrm{L}$ of the final supernatant was analyzed in a plate-reading fluorimeter (Molecular Devices Gemini XPS or Gemini EM; excitation 595 nm; cutoff 610 nm; emission 615 nm).

\section{ACKNOWLEDGEMENTS}

We are grateful to Drs. M. Ailion, J. Bai, R. Baker, J. Cattin, S. Hoppins, I. Topalidou, and M. Zick for helpful advice and critical comments on the manuscript; C. Barlowe, C. Boone, and D. Waugh for antibodies and strains, D. Baker and the University of Washington Institute for Protein Design for computational resources, and D. Beacham (Molecular Probes/Thermo Fisher) for gifts of fluorescent reagents. These studies were supported by NIH/NIGMS R01 GM077349 and the University of Washington (AM), NIH/NIGMS T32 GM007270 (UN), NIH MARC T34 GM083883 (BD) and Medical Research Council MC_UP_1201/10 (EM). 


\section{REFERENCES}

715

Antonny, B., D. Madden, S. Hamamoto, L. Orci, and R. Schekman. 2001. Dynamics of the COPII coat with GTP and stable analogues. Nat Cell Biol. 3:531-537.

Arnold, M. G., P. Adhikari, B. Kang, and H. Xu 徐昊. 2017. Munc18a clusters SNARE-bearing liposomes prior to trans-SNARE zippering. Biochem J. 474:3339-3354.

Baker, D., L. Hicke, M. Rexach, M. Schleyer, and R. Schekman. 1988. Reconstitution of SEC gene productdependent intercompartmental protein transport. Cell. 54:335-344.

Baker, R. W., and F. M. Hughson. 2016. Chaperoning SNARE assembly and disassembly. Nat Rev Mol Cell Biol. 17:465-479.

Baker, R. W., P. D. Jeffrey, M. Zick, B. P. Phillips, W. T. Wickner, and F. M. Hughson. 2015. A direct role for the Sec1/Munc18-family protein Vps33 as a template for SNARE assembly. Science. 349:11111114.

Ballew, N., Y. Liu, and C. Barlowe. 2005. A Rab requirement is not bypassed in SLY1-20 suppression. Mol Biol Cell. 16:1839-1849.

Banta, L. M., T. A. Vida, P. K. Herman, and S. D. Emr. 1990. Characterization of yeast Vps33p, a protein required for vacuolar protein sorting and vacuole biogenesis. Mol Cell Biol. 10:4638-4649.

Bensen, E. S., B. G. Yeung, and G. S. Payne. 2001. Ric1p and the Ypt6p GTPase function in a common pathway required for localization of trans-Golgi network membrane proteins. Mol Biol Cell. 12:1326.

Bigay, J., and B. Antonny. 2012. Curvature, lipid packing, and electrostatics of membrane organelles: defining cellular territories in determining specificity. Dev Cell. 23:886-895.

Bigay, J., J. F. Casella, G. Drin, B. Mesmin, and B. Antonny. 2005. ArfGAP1 responds to membrane curvature through the folding of a lipid packing sensor motif. EMBO J. 24:2244-2253.

Bracher, A., and W. Weissenhorn. 2002. Structural basis for the Golgi membrane recruitment of Sly1p by Sed5p. EMBO J. 21:6114-6124.

Breslow, D. K., D. M. Cameron, S. R. Collins, M. Schuldiner, J. Stewart-Ornstein, H. W. Newman, S. Braun, H. D. Madhani, N. J. Krogan, and J. S. Weissman. 2008. A comprehensive strategy enabling highresolution functional analysis of the yeast genome. Nat Methods. 5:711-718.

Burd, C. G., M. Peterson, C. R. Cowles, and S. D. Emr. 1997. A novel Sec18p/NSF-dependent complex required for Golgi-to-endosome transport in yeast. Mol Biol Cell. 8:1089-1104.

Busch, D. J., J. R. Houser, C. C. Hayden, M. B. Sherman, E. M. Lafer, and J. C. Stachowiak. 2015. Intrinsically disordered proteins drive membrane curvature. Nat Commun. 6:7875.

Cabrera, M., L. Langemeyer, M. Mari, R. Rethmeier, I. Orban, A. Perz, C. Brocker, J. Griffith, D. Klose, H. J. Steinhoff, F. Reggiori, S. Engelbrecht-Vandre, and C. Ungermann. 2010. Phosphorylation of a membrane curvature-sensing motif switches function of the HOPS subunit Vps41 in membrane tethering. J Cell Biol. 191:845-859.

Cao, X., and C. Barlowe. 2000. Asymmetric requirements for a Rab GTPase and SNARE proteins in fusion of COPII vesicles with acceptor membranes. J Cell Biol. 149:55-66.

Carr, C. M., and J. Rizo. 2010. At the junction of SNARE and SM protein function. Curr Opin Cell Biol. 22:488-495.

Chen, P. S., T. Y. T. Toribara, and H. Warner. 1956. Microdetermination of phosphorus. Analytical chemistry. 28:1756-1758.

Cheung, P. Y., C. Limouse, H. Mabuchi, and S. R. Pfeffer. 2015. Protein flexibility is required for vesicle tethering at the Golgi. Elife. 4

Cheung, P. Y., and S. R. Pfeffer. 2016. Transport Vesicle Tethering at the Trans Golgi Network: Coiled Coil Proteins in Action. Front Cell Dev Biol. 4:18.

Chou, H. T., D. Dukovski, M. G. Chambers, K. M. Reinisch, and T. Walz. 2016. CATCHR, HOPS and CORVET tethering complexes share a similar architecture. Nat Struct Mol Biol. 23:761-763. 
Cowles, C. R., S. D. Emr, and B. F. Horazdovsky. 1994. Mutations in the VPS45 gene, a SEC1 homologue, result in vacuolar protein sorting defects and accumulation of membrane vesicles. J Cell Sci. 107:3449-3459.

D’Agostino, M., H. J. Risselada, A. Lürick, C. Ungermann, and A. Mayer. 2017. A tethering complex drives the terminal stage of SNARE-dependent membrane fusion. Nature.

Dascher, C., R. Ossig, D. Gallwitz, and H. D. Schmitt. 1991. Identification and structure of four yeast genes (SLY) that are able to suppress the functional loss of YPT1, a member of the RAS superfamily. Mol Cell Biol. 11:872-885.

Demircioglu, F. E., P. Burkhardt, and D. Fasshauer. 2014. The SM protein Sly1 accelerates assembly of the ER-Golgi SNARE complex. Proc Natl Acad Sci U S A. 111:13828-13833.

Drin, G., and B. Antonny. 2010. Amphipathic helices and membrane curvature. FEBS Lett. 584:1840-1847.

Drin, G., V. Morello, J. F. Casella, P. Gounon, and B. Antonny. 2008. Asymmetric tethering of flat and curved lipid membranes by a golgin. Science. 320:670-673.

Duan, M., G. Gao, D. Banfield, T. Takenaka, and A. J. Merz. submitted. Golgi SM protein Sly1 promotes productive trans-SNARE complex assembly through multiple mechanisms.

Dunham, M. J., M. R. Gartenberg, and G. W. Brown. 2015. Methods in yeast genetics and genomics, 2015 edition: a CSHL course manual.

Evers, T. H., E. M. van Dongen, A. C. Faesen, E. W. Meijer, and M. Merkx. 2006. Quantitative understanding of the energy transfer between fluorescent proteins connected via flexible peptide linkers. Biochemistry. 45:13183-13192.

Furukawa, N., and J. Mima. 2014. Multiple and distinct strategies of yeast SNAREs to confer the specificity of membrane fusion. Sci Rep. 4:4277.

Gillingham, A. K., and S. Munro. 2019. Transport carrier tethering - how vesicles are captured by organelles. Curr Opin Cell Biol. 59:140-146.

Gillingham, A. K. 2018. At the ends of their tethers! How coiled-coil proteins capture vesicles at the Golgi. Biochemical Society Transactions. 46:43-50.

Grabowski, R., and D. Gallwitz. 1997. High-affinity binding of the yeast cis-Golgi t-SNARE, Sed5p, to wildtype and mutant Sly1p, a modulator of transport vesicle docking. FEBS Lett. 411:169-172.

Grote, E., C. M. Carr, and P. J. Novick. 2000. Ordering the final events in yeast exocytosis. J Cell Biol. 151:439-452.

Ha, J. Y., H. T. Chou, D. Ungar, C. K. Yip, T. Walz, and F. M. Hughson. 2016. Molecular architecture of the complete COG tethering complex. Nat Struct Mol Biol. 23:758-760.

Haas, A., and W. Wickner. 1996. Homotypic vacuole fusion requires Sec17p (yeast alpha-SNAP) and Sec18p (yeast NSF). EMBO J. 15:3296-3305.

Jiao, J., M. He, S. A. Port, R. W. Baker, Y. Xu, H. Qu, Y. Xiong, Y. Wang, H. Jin, T. J. Eisemann, F. M. Hughson, and Y. Zhang. 2018. Munc18-1 catalyzes neuronal SNARE assembly by templating SNARE association. Elife. 7

Jun, Y., and W. Wickner. 2019. Sec17 ( $\alpha$-SNAP) and Sec18 (NSF) restrict membrane fusion to R-SNAREs, Q-SNAREs, and SM proteins from identical compartments. Proceedings of the National Academy of Sciences. 201913985.

Kapust, R. B., K. M. Routzahn, and D. S. Waugh. 2002. Processive degradation of nascent polypeptides, triggered by tandem AGA codons, limits the accumulation of recombinant tobacco etch virus protease in Escherichia coli BL21(DE3). Protein Expr Purif. 24:61-70.

Klemm, R. W., C. S. Ejsing, M. A. Surma, H. J. Kaiser, M. J. Gerl, J. L. Sampaio, Q. de Robillard, C. Ferguson, T. J. Proszynski, A. Shevchenko, and K. Simons. 2009. Segregation of sphingolipids and sterols during formation of secretory vesicles at the trans-Golgi network. J Cell Biol. 185:601-612.

Kosodo, Y., Y. Noda, and K. Yoda. 1998. Protein-protein interactions of the yeast Golgi t-SNARE Sed5 protein distinct from its neural plasma membrane cognate syntaxin 1. Biochem Biophys Res Commun. 250:212-216. 
Kraynack, B. A., A. Chan, E. Rosenthal, M. Essid, B. Umansky, M. G. Waters, and H. D. Schmitt. 2005. Dsl1p, Tip20p, and the novel Dsl3(Sec39) protein are required for the stability of the Q/t-SNARE complex at the endoplasmic reticulum in yeast. Mol Biol Cell. 16:3963-3977.

Laufman, O., A. Kedan, W. Hong, and S. Lev. 2009. Direct interaction between the COG complex and the SM protein, Sly1, is required for Golgi SNARE pairing. EMBO J. 28:2006-2017.

Leaver-Fay, A., M. Tyka, S. M. Lewis, O. F. Lange, J. Thompson, R. Jacak, K. Kaufman, P. D. Renfrew, C. A. Smith, W. Sheffler, I. W. Davis, S. Cooper, A. Treuille, D. J. Mandell, F. Richter, Y. E. Ban, S. J. Fleishman, J. E. Corn, D. E. Kim, S. Lyskov, M. Berrondo, S. Mentzer, Z. Popović, J. J. Havranek, J. Karanicolas, R. Das, J. Meiler, T. Kortemme, J. J. Gray, B. Kuhlman, D. Baker, and P. Bradley. 2011. ROSETTA3: an object-oriented software suite for the simulation and design of macromolecules. Methods Enzymol. 487:545-574.

Li, Y., D. Gallwitz, and R. Peng. 2005. Structure-based functional analysis reveals a role for the SM protein Sly1p in retrograde transport to the endoplasmic reticulum. Mol Biol Cell. 16:3951-3962.

Li, Y., H. D. Schmitt, D. Gallwitz, and R. W. Peng. 2007. Mutations of the SM protein Sly1 resulting in bypass of GTPase requirement in vesicular transport are confined to a short helical region. FEBS Lett. 581:5698-5702.

Lo, S. Y., C. L. Brett, R. L. Plemel, M. Vignali, S. Fields, T. Gonen, and A. J. Merz. 2012. Intrinsic tethering activity of endosomal Rab proteins. Nat Struct Mol Biol. 19:40-47.

Lobingier, B. T., D. P. Nickerson, S. Y. Lo, and A. J. Merz. 2014. SM proteins Sly1 and Vps33 co-assemble with Sec17 and SNARE complexes to oppose SNARE disassembly by Sec18. Elife. 3:e02272.

Lupashin, V. V., S. Hamamoto, and R. W. Schekman. 1996. Biochemical requirements for the targeting and fusion of ER-derived transport vesicles with purified yeast Golgi membranes. J Cell Biol. 132:277-289.

Ma, C., L. Su, A. B. Seven, Y. Xu, and J. Rizo. 2013. Reconstitution of the vital functions of Munc18 and Munc13 in neurotransmitter release. Science. 339:421-425.

Magdeleine, M., R. Gautier, P. Gounon, H. Barelli, S. Vanni, and B. Antonny. 2016. A filter at the entrance of the Golgi that selects vesicles according to size and bulk lipid composition. Elife. 5

Matsuoka, K., L. Orci, M. Amherdt, S. Y. Bednarek, S. Hamamoto, R. Schekman, and T. Yeung. 1998. COPII-coated vesicle formation reconstituted with purified coat proteins and chemically defined liposomes. Cell. 93:263-275.

Mi, H., A. Muruganujan, D. Ebert, X. Huang, and P. D. Thomas. 2019. PANTHER version 14: more genomes, a new PANTHER GO-slim and improvements in enrichment analysis tools. Nucleic Acids Res. 47:D419-D426.

Morgera, F., M. R. Sallah, M. L. Dubuke, P. Gandhi, D. N. Brewer, C. M. Carr, and M. Munson. 2012. Regulation of exocytosis by the exocyst subunit Sec6 and the SM protein Sec1. Mol Biol Cell. 23:337-346.

Murray, D. H., M. Jahnel, J. Lauer, M. J. Avellaneda, N. Brouilly, A. Cezanne, H. Morales-Navarrete, E. D. Perini, C. Ferguson, A. N. Lupas, Y. Kalaidzidis, R. G. Parton, S. W. Grill, and M. Zerial. 2016. An endosomal tether undergoes an entropic collapse to bring vesicles together. Nature. 537:107111.

Novick, P., R. Schekman, P. Novick, C. Field, and R. Schekman. 1979. Secretion and cell-surface growth are blocked in a temperature-sensitive mutant of Saccharomyces cerevisiae.

Identification of 23 complementation groups required for post-translational events in the yeast secretory pathway. Proc Natl Acad Sci U S A Cell. 76:1858-1862.

Ossig, R., C. Dascher, H. H. Trepte, H. D. Schmitt, and D. Gallwitz. 1991. The yeast SLY gene products, suppressors of defects in the essential GTP-binding Ypt1 protein, may act in endoplasmic reticulum-to-Golgi transport. Mol Cell Biol. 11:2980-2993.

Ossig, R., W. Laufer, H. D. Schmitt, and D. Gallwitz. 1995. Functionality and specific membrane localization of transport GTPases carrying C-terminal membrane anchors of synaptobrevin-like proteins. Embo J. 14:3645-3653. 
Patterson, J. T. 1932. A New Type of Mottled-Eyed Drosophila Due to an Unstable Translocation. Genetics. 17:38-59.

Peng, R., and D. Gallwitz. 2002. Sly1 protein bound to Golgi syntaxin Sed5p allows assembly and contributes to specificity of SNARE fusion complexes. J Cell Biol. 157:645-655.

Peng, R., and D. Gallwitz. 2004. Multiple SNARE interactions of an SM protein: Sed5p/Sly1p binding is dispensable for transport. EMBO J. 23:3939-3949.

Peterson, M. R., C. G. Burd, and S. D. Emr. 1999. Vac1p coordinates Rab and phosphatidylinositol 3kinase signaling in Vps45p-dependent vesicle docking/fusion at the endosome. Curr Biol. 9:159162.

Piper, R. C., E. A. Whitters, and T. H. Stevens. 1994. Yeast Vps45p is a Sec1p-like protein required for the consumption of vacuole-targeted, post-Golgi transport vesicles. Eur J Cell Biol. 65:305-318.

Rahajeng, J., S. Caplan, and N. Naslavsky. 2010. Common and distinct roles for the binding partners Rabenosyn-5 and Vps45 in the regulation of endocytic trafficking in mammalian cells. Exp Cell Res. 316:859-874.

Reilly, B. A., B. A. Kraynack, S. M. VanRheenen, and M. G. Waters. 2001. Golgi-to-endoplasmic reticulum (ER) retrograde traffic in yeast requires Dsl1p, a component of the ER target site that interacts with a COPI coat subunit. Mol Biol Cell. 12:3783-3796.

Ren, Y., C. K. Yip, A. Tripathi, D. Huie, P. D. Jeffrey, T. Walz, and F. M. Hughson. 2009. A structure-based mechanism for vesicle capture by the multisubunit tethering complex Dsl1. Cell. 139:1119-1129.

Rieder, S. E., and S. D. Emr. 1997. A novel RING finger protein complex essential for a late step in protein transport to the yeast vacuole. Mol Biol Cell. 8:2307-2327.

Rizo, J., and T. C. Sudhof. 2012. The membrane fusion enigma: SNAREs, Sec1/Munc18 proteins, and their accomplices--guilty as charged? Annu Rev Cell Dev Biol. 28:279-308.

Rossi, G., D. Lepore, L. Kenner, A. B. Czuchra, M. Plooster, A. Frost, M. Munson, and P. Brennwald. 2020. Exocyst structural changes associated with activation of tethering downstream of Rho/Cdc42 GTPases. J Cell Biol. 219

Ruohola, H., A. K. Kabcenell, and S. Ferro-Novick. 1988. Reconstitution of protein transport from the endoplasmic reticulum to the Golgi complex in yeast: the acceptor Golgi compartment is defective in the sec23 mutant. J Cell Biol. 107:1465-1476.

Sacher, M., Y. Jiang, J. Barrowman, A. Scarpa, J. Burston, L. Zhang, D. Schieltz, J. R. Yates, 3rd, H. Abeliovich, and S. Ferro-Novick. 1998. TRAPP, a highly conserved novel complex on the cis-Golgi that mediates vesicle docking and fusion. Embo J. 17:2494-2503.

Sapperstein, S. K., V. V. Lupashin, H. D. Schmitt, and M. G. Waters. 1996. Assembly of the ER to Golgi SNARE complex requires Uso1p. J Cell Biol. 132:755-767.

Schneiter, R., B. Brügger, R. Sandhoff, G. Zellnig, A. Leber, M. Lampl, K. Athenstaedt, C. Hrastnik, S. Eder, G. Daum, F. Paltauf, F. T. Wieland, and S. D. Kohlwein. 1999. Electrospray ionization tandem mass spectrometry (ESI-MS/MS) analysis of the lipid molecular species composition of yeast subcellular membranes reveals acyl chain-based sorting/remodeling of distinct molecular species en route to the plasma membrane. J Cell Biol. 146:741-754.

Schwartz, M. L., and A. J. Merz. 2009. Capture and release of partially zipped trans-SNARE complexes on intact organelles. J Cell Biol. 185:535-549.

Schwartz, M. L., D. P. Nickerson, B. T. Lobingier, R. L. Plemel, M. Duan, C. G. Angers, M. Zick, and A. J. Merz. 2017. Sec17 ( $\alpha$-SNAP) and an SM-tethering complex regulate the outcome of SNARE zippering in vitro and in vivo. Elife. 6

Sevrioukov, E. A., J. P. He, N. Moghrabi, A. Sunio, and H. Kramer. 1999. A role for the deep orange and carnation eye color genes in lysosomal delivery in Drosophila. Mol Cell. 4:479-486.

Sheffield, P., S. Garrard, and Z. Derewenda. 1999. Overcoming expression and purification problems of RhoGDI using a family of "parallel" expression vectors. Protein Expr Purif. 15:34-39.

910 Sogaard, M., K. Tani, R. R. Ye, S. Geromanos, P. Tempst, T. Kirchhausen, J. E. Rothman, and T. Sollner. 1994. A rab protein is required for the assembly of SNARE complexes in the docking of transport vesicles. Cell. 78:937-948. 
Studier, F. W. 2005. Protein production by auto-induction in high-density shaking cultures. Protein Expression and Purification. 41:207-234.

915 Sudhof, T. C., and J. E. Rothman. 2009. Membrane fusion: grappling with SNARE and SM proteins. Science. 323:474-477.

Tall, G. G., H. Hama, D. B. DeWald, and B. F. Horazdovsky. 1999. The phosphatidylinositol 3-phosphate binding protein Vac1p interacts with a Rab GTPase and a Sec1p homologue to facilitate vesiclemediated vacuolar protein sorting. Mol Biol Cell. 10:1873-1889.

Tareste, D., J. Shen, T. J. Melia, and J. E. Rothman. 2008. SNAREpin/Munc18 promotes adhesion and fusion of large vesicles to giant membranes. Proc Natl Acad Sci U S A. 105:2380-2385.

The Gene Ontology Consortium. 2019. The Gene Ontology Resource: 20 years and still GOing strong. Nucleic Acids Res. 47:D330-D338.

Tong, A. H., and C. Boone. 2005. Synthetic Genetic Array Analysis in Saccharomyces cerevisiae. Methods Mol Biol. 313:171-192.

van Leeuwen, J., C. Pons, J. C. Mellor, T. N. Yamaguchi, H. Friesen, J. Koschwanez, M. M. Ušaj, M. Pechlaner, M. Takar, M. Ušaj, B. VanderSluis, K. Andrusiak, P. Bansal, A. Baryshnikova, C. E. Boone, J. Cao, A. Cote, M. Gebbia, G. Horecka, I. Horecka, E. Kuzmin, N. Legro, W. Liang, N. van Lieshout, M. McNee, B. J. San Luis, F. Shaeri, E. Shuteriqi, S. Sun, L. Yang, J. Y. Youn, M. Yuen, M. Costanzo, A. C. Gingras, P. Aloy, C. Oostenbrink, A. Murray, T. R. Graham, C. L. Myers, B. J. Andrews, F. P. Roth, and C. Boone. 2016. Exploring genetic suppression interactions on a global scale. Science. 354

VanRheenen, S. M., X. Cao, V. V. Lupashin, C. Barlowe, and M. G. Waters. 1998. Sec35p, a novel peripheral membrane protein, is required for ER to Golgi vesicle docking. J Cell Biol. 141:1107-1119.

VanRheenen, S. M., X. Cao, S. K. Sapperstein, E. C. Chiang, V. V. Lupashin, C. Barlowe, and M. G. Waters. 1999. Sec34p, a protein required for vesicle tethering to the yeast Golgi apparatus, is in a complex with Sec35p. J Cell Biol. 147:729-742.

Vanrheenen, S. M., B. A. Reilly, S. J. Chamberlain, and M. G. Waters. 2001. Dsl1p, an essential protein required for membrane traffic at the endoplasmic reticulum/Golgi interface in yeast. Traffic. 2:212-231.

Verhage, M., A. S. Maia, J. J. Plomp, A. B. Brussaard, J. H. Heeroma, H. Vermeer, R. F. Toonen, R. E. Hammer, T. K. van den Berg, M. Missler, H. J. Geuze, and T. C. Sudhof. 2000. Synaptic assembly of the brain in the absence of neurotransmitter secretion. Science. 287:864-869.

Wu, M. N., J. T. Littleton, M. A. Bhat, A. Prokop, and H. J. Bellen. 1998. ROP, the Drosophila Sec1 homolog, interacts with syntaxin and regulates neurotransmitter release in a dosage-dependent manner. EMBO J. 17:127-139.

Xu, H., Y. Jun, J. Thompson, J. Yates, and W. Wickner. 2010. HOPS prevents the disassembly of transSNARE complexes by Sec17p/Sec18p during membrane fusion. EMBO J. 29:1948-1960.

Yamaguchi, T., I. Dulubova, S. W. Min, X. Chen, J. Rizo, and T. C. Südhof. 2002. Sly1 binds to Golgi and ER syntaxins via a conserved N-terminal peptide motif. Dev Cell. 2:295-305.

Yu, H., S. S. Rathore, C. Shen, Y. Liu, Y. Ouyang, M. H. Stowell, and J. Shen. 2015. Reconstituting Intracellular Vesicle Fusion Reactions: The Essential Role of Macromolecular Crowding. J Am Chem Soc. 137:12873-12883.

Zucchi, P. C., and M. Zick. 2011. Membrane fusion catalyzed by a Rab, SNAREs, and SNARE chaperones is accompanied by enhanced permeability to small molecules and by lysis. Mol Biol Cell. 22:46354646. 


\section{SUPPLEMENTARY MATERIAL}

A. Sly1-2f

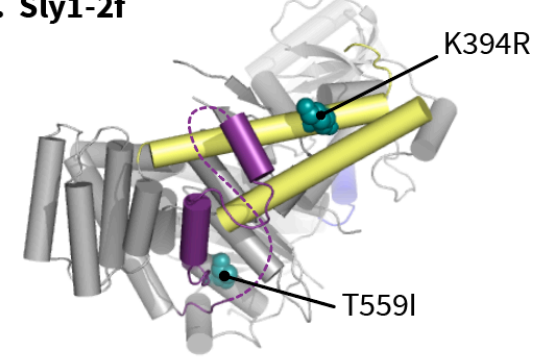

C. Sly1-3r

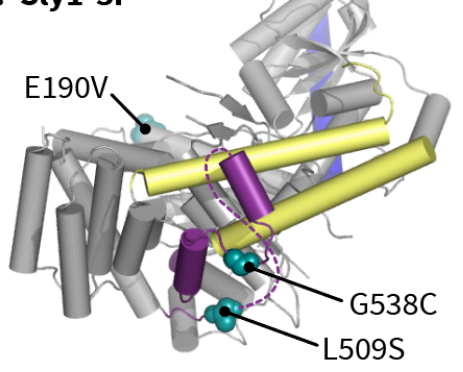

B. Sly1-2m

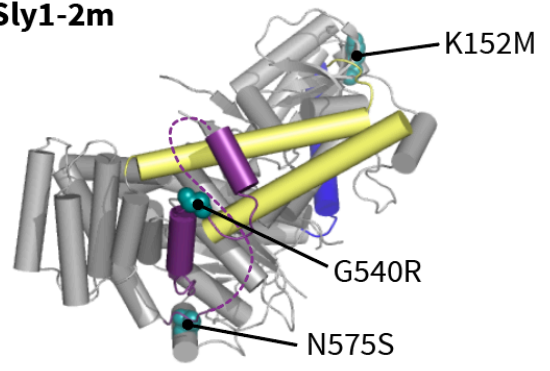

D. Sly1-4h

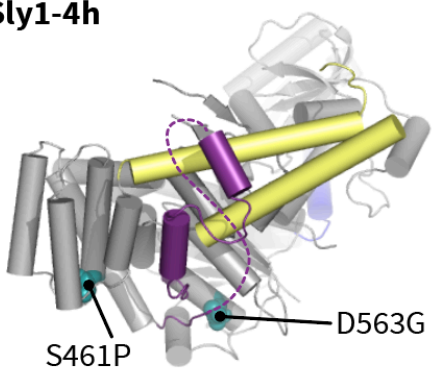

D.

genome: uso1 $\triangle:: K A N$

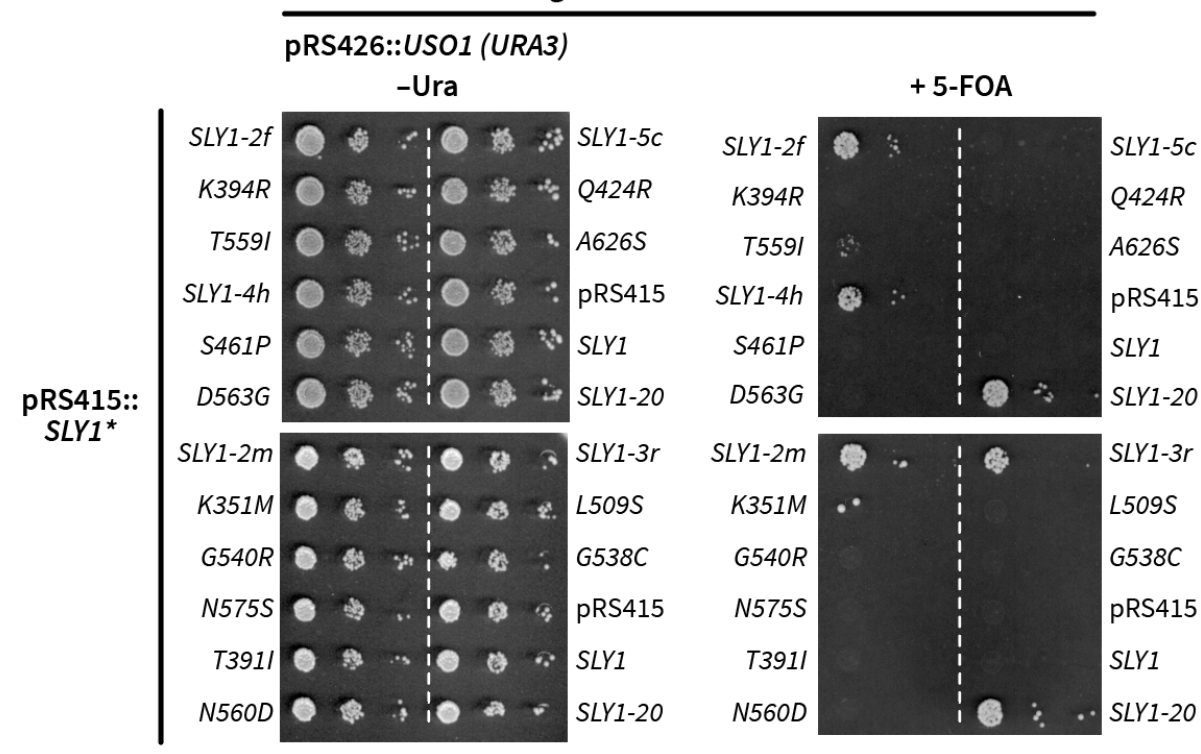

Supplementary Fig. S1. Some $S L Y 1$ alleles require multiple substitutions to suppress the lethal uso1 $\Delta$ phenotype. A-D, Locations of amino acid substitutions in four representative $S L Y 1$ alleles recovered in our screen. E, Growth phenotypes show that most single substitutions are unable to suppress the total absence of Uso1. Many of the same single mutants suppress deficiency of Ypt1 (see Supplementary Table I). The multisite allele $S L Y 1-5 c$, although retrieved in our primary screen, was unable to suppress the uso1 $\Delta$ allele in secondary screening. 


\section{Supplementary Table 1. Selected SLY1 mutants and their growth phenotypes.}

\begin{tabular}{|c|c|c|c|c|}
\hline $\begin{array}{l}\text { Sly1* substitution } \\
\text { (protein) }\end{array}$ & $\begin{array}{l}\text { SLY1* allele } \\
\text { (nucleotide) }\end{array}$ & $\begin{array}{c}\text { Complements } \\
{\text { sly } 1 \Delta^{1}}^{1}\end{array}$ & $\begin{array}{c}\text { Bypasses } \\
\text { uso1 }^{1}\end{array}$ & Bypasses ypt1-3 ${ }^{2}$ \\
\hline E4D & $\mathrm{G} 12 \mathrm{C}$ & ++ & - & - \\
\hline V82L & G244C & ++ & - & - \\
\hline E190V & A569T & ++ & - & - \\
\hline K351M & $\mathrm{A} 1052 \mathrm{~T}$ & ++ & - & - \\
\hline T391I & $\mathrm{C} 1172 \mathrm{~T}$ & ++ & - & - \\
\hline K394R & A1181G & ++ & - & - \\
\hline T418I & $\mathrm{C} 1253 \mathrm{~T}$ & ++ & - & - \\
\hline Q424R & A1271G & ++ & - & - \\
\hline S461P & $\mathrm{T} 1381 \mathrm{C}$ & ++ & - & - \\
\hline L509S & T1526C & ++ & - & ++ \\
\hline T531I = Sly1-15 & C1592T & ++ & ++ & ++ \\
\hline E532K = Sly1-20 & G1594A & ++ & ++ & ++ \\
\hline G538C & G1612T & ++ & $+/-$ & ++ \\
\hline G540R & G1618A & ++ & $+/-$ & ++ \\
\hline T559I & $\mathrm{C} 1676 \mathrm{~T}$ & ++ & + & ++ \\
\hline N560D & A1678G & ++ & - & ++ \\
\hline D563G & A1688G & ++ & $+/-$ & ++ \\
\hline N575S & A1724G & ++ & - & ++ \\
\hline A626S & G1876T & ++ & - & - \\
\hline $\mathrm{E} 648 \mathrm{~A}$ & A1943C & ++ & - & - \\
\hline $\begin{array}{c}\text { Sly1-pa21 } \\
\text { L542S I543T I546T } \\
\text { L549S L550S }\end{array}$ & $\begin{array}{l}\text { See Materials } \\
\text { \& Methods }\end{array}$ & - & - & - \\
\hline $\begin{array}{c}\text { Sly1-20-pa21 } \\
\text { T531I L542S I543T } \\
\text { I546T L549S L550S }\end{array}$ & $\begin{array}{l}\text { See Materials } \\
\& \text { Methods }\end{array}$ & ++ & - & - \\
\hline \multicolumn{5}{|c|}{$\begin{array}{l}{ }^{1} \text { Growth of sly } 1 \triangle \text { (or uso1 } 1 \text { ) cells, bearing the indicated SLY1 allele on a LEU2-marked plasmid and wild-type } \\
S L Y 1 \text { (or USO1) on a counter-selectable URA3-marked balancer plasmid was assessed following ejection of the } \\
\text { balancer plasmid on 5-FOA plates incubated at } 30^{\circ} \mathrm{C} \text { for } 2 \text { days. } \\
{ }^{2} \text { Growth of } y p t 1-3 \text { cells bearing the indicated alleles of } S L Y 1 \text { on a } L E U 2 \text {-marked plasmid and wild-type YPT1 on a } \\
\text { counter-selectable URA3-marked balancer plasmid was assessed following ejection of the balancer plasmid on } \\
5 \text {-FOA plates incubated at } 37^{\circ} \mathrm{C} \text { for } 2-3 \text { days. }\end{array}$} \\
\hline
\end{tabular}


bioRxiv preprint doi: https://doi.org/10.1101/2020.01.16.906719; this version posted January 16,2020 . The copyright holder for this preprint (which was not certified by peer review) is the author/funder, who has granted bioRxiv a license to display the preprint in perpetuity. It is made available under aCC-BY-NC-ND 4.0 International license.

\section{Supplementary Table 2. SLY1 "loopless" mutants and their growth phenotypes.}

\begin{tabular}{|c|c|c|c|c|c|}
\hline Sly1 mutant & $\begin{array}{l}\text { Residues } \\
\text { excised }\end{array}$ & $\begin{array}{l}\text { Synthetic linker } \\
\text { (AA/dna) }\end{array}$ & $\begin{array}{c}\text { Complements } \\
{\text { sly } 1 \Delta^{1}}^{1}\end{array}$ & $\begin{array}{c}\text { Bypasses } \\
\text { uso1 }^{1}\end{array}$ & $\begin{array}{c}\text { Bypasses } \\
\text { ypt1-3 }^{2}\end{array}$ \\
\hline Sly1 (wt) & - & - & ++ & - & - \\
\hline Sly1-0_1 & $\Delta 500-558$ & $\begin{array}{c}\text { WADKGDGGVT } \\
\text { tgggctgataaaggtgatggtggtgtt }\end{array}$ & + & - & - \\
\hline $\begin{aligned} & \text { Sly } 1-0 \_2 \\
= & \text { Sly1 } 1 \Delta \text { loop }\end{aligned}$ & $\Delta 500-558$ & $\begin{array}{c}\text { WAKKGDGGT } \\
\text { tgggctaaaaaaggtgatggtggtact }\end{array}$ & + & - & - \\
\hline Sly1-0_3 & $\Delta 500-558$ & $\begin{array}{c}\text { WAKKGDGGV } \\
\text { tgggctaaaaaaggtgatggtggtgtt }\end{array}$ & + & - & - \\
\hline Sly1-0_4 & $\Delta 500-558$ & $\begin{array}{l}\text { WAKKSADGAPT } \\
\text { tgggctaaaaaatctgctgatggtgctccaact }\end{array}$ & + & - & - \\
\hline Sly1-0_5 & $\Delta 500-558$ & $\begin{array}{c}\text { YAKLSADGAPV } \\
\text { tatgctaaattgtctgctgatggtgctccagtt }\end{array}$ & $+/-$ & - & - \\
\hline Sly1-0_6 & $\Delta 500-558$ & $\begin{array}{c}\text { WAKAAGGDNPT } \\
\text { tgggctaaagctgctggtggtgataatccaact }\end{array}$ & + & - & - \\
\hline Sly1-0_7 & $\Delta 500-558$ & $\begin{array}{c}\text { WAKAAGGTHPT } \\
\text { tgggctaaagctgctggtggtactcatccaact }\end{array}$ & + & - & - \\
\hline Sly1-0_8 & $\Delta 500-558$ & $\begin{array}{c}\text { YAKASSEATGPT } \\
\text { tatgctaaagcttcttctgaagctactggtccaact }\end{array}$ & + & - & - \\
\hline Sly1-0_9 & $\Delta 500-558$ & $\begin{array}{c}\text { YADQQGTNAGPV } \\
\text { tatgctgatcaacaaggtactaatgctggtccagtt }\end{array}$ & + & - & - \\
\hline Sly1-0_10 & $\Delta 500-558$ & $\begin{array}{c}\text { YNNGAGTGGPT } \\
\text { tataataatggtgctggtactggtggtccaact }\end{array}$ & + & - & - \\
\hline Sly1-3_2 & $\Delta 497-560$ & $\begin{array}{c}\text { QEATSKSGGTGPTVA } \\
\text { caagaagctacttctaaatctggtggtactggtccaac } \\
\text { tgttgct }\end{array}$ & - & - & - \\
\hline Sly1-3_5 & $\Delta 497-560$ & $\begin{array}{c}\text { QQTYDNSGKDAAPTVC } \\
\text { caacaaacttatgataattctggtaaagatgctgctcc } \\
\text { aactgtttgt }\end{array}$ & - & - & - \\
\hline \multicolumn{6}{|c|}{$\begin{array}{l}{ }^{1} \text { Growth of } s / y 1 \triangle \text { (or uso1A) cells, bearing the indicated allele on a } L E U 2 \text {-marked plasmid and wild-type } S L Y 1 \text { (or } \\
\text { USO1) on a counter-selectable URA3-marked balancer plasmid was assessed following ejection of the balancer } \\
\text { plasmid on 5-FOA plates incubated at } 30^{\circ} \mathrm{C} \text { for } 2-3 \text { days. } \\
{ }^{2} \text { Growth of ypt1-3 cells bearing the indicated alleles of } S L Y 1 \text { on a } L E U 2 \text {-marked plasmid and wild-type YPT1 on a } \\
\text { counter-selectable URA3-marked balancer plasmid was assessed following ejection of the balancer plasmid on } \\
5 \text {-FOA plates incubated at } 37^{\circ} \mathrm{C} \text { for } 2-3 \text { days. }\end{array}$} \\
\hline
\end{tabular}




\section{Supplementary Table 3. SLY1 “loopless" chimeras with N-terminal Loop attachments, and their growth phenotypes.}

\begin{tabular}{|c|c|c|c|c|c|}
\hline Sly1 mutant & $\begin{array}{l}\text { Residues } \\
\text { excised }\end{array}$ & $\begin{array}{l}\text { Synthetic linker } \\
\text { (AA/dna) }\end{array}$ & $\begin{array}{l}\text { Complements } \\
\text { sly1 } 1 \Delta^{1}\end{array}$ & $\begin{array}{l}\text { Bypasses } \\
\text { uso1 }^{1}\end{array}$ & $\begin{array}{c}\text { Bypasses } \\
\text { ypt1-3 }^{2}\end{array}$ \\
\hline Sly1 (wt) & - & - & ++ & - & - \\
\hline $\begin{aligned} & \text { Sly1-0_2 } \\
= & \text { Sly1 } \Delta \text { loop }\end{aligned}$ & $\Delta 500-558$ & $\begin{array}{c}\text { WAKKGDGGT } \\
\text { tgggctaaaaaaggtgatggtggtact }\end{array}$ & + & - & - \\
\hline $\begin{array}{l}\text { loop- } \\
\text { Sly1 } 1 \text { loop }\end{array}$ & $\Delta 500-558$ & $\begin{array}{l}\text { Sly1 } 1 \text { loop, with Sly1 aa 502-558- } \\
\text { GGSGGSG appended to N-terminus }\end{array}$ & ++ & - & - \\
\hline $\begin{array}{l}\alpha 20-\alpha 21- \\
\text { Sly1 } 1 \text { loop }\end{array}$ & $\Delta 500-558$ & $\begin{array}{l}\text { Sly1 } 1 \text { loop, with Sly1 aa 526-558- } \\
\text { GGSGGSG appended to N-terminus }\end{array}$ & ++ & - & - \\
\hline $\begin{array}{c}\alpha 21- \\
\text { Sly1 } 1 \Delta \text { loop }\end{array}$ & $\Delta 500-558$ & $\begin{array}{l}\text { Sly1 } 1 \text { loop, with Sly1 aa 540-558- } \\
\text { GGSGGSG appended to N-terminus }\end{array}$ & ++ & - & - \\
\hline $\begin{array}{l}\operatorname{loop}(p \alpha 21)- \\
\text { Sly } 1 \Delta \text { loop }\end{array}$ & $\Delta 500-558$ & $\begin{array}{l}\text { Sly1 } 1 \text { loop, with Sly1 aa } 502-558- \\
\text { GGSGGSG appended to N-terminus; } \\
\text { appended sequence contains pa21 } \\
\text { substitutions }\end{array}$ & - & - & - \\
\hline $\begin{array}{l}\alpha 20-p \alpha 21- \\
\text { Sly1 } 1 \text { loop }\end{array}$ & $\Delta 500-558$ & $\begin{array}{l}\text { Sly1 } 1 \text { loop, with Sly1 aa } 526-558- \\
\text { GGSGGSG appended to N-terminus; } \\
\text { appended sequence contains pa21 } \\
\text { substitutions }\end{array}$ & - & - & - \\
\hline $\begin{array}{l}\text { pa21- } \\
\text { Sly1 } 1 \text { loop }\end{array}$ & $\Delta 500-558$ & $\begin{array}{l}\text { Sly1 } 1 \text { loop, with Sly1 aa } 540-558- \\
\text { GGSGGSG appended to N-terminus; } \\
\text { appended sequence contains pa21 } \\
\text { substitutions }\end{array}$ & ++ & - & - \\
\hline \multicolumn{6}{|c|}{$\begin{array}{l}{ }^{1} \text { Growth of sly } 1 \triangle \text { (or uso1D) cells, bearing the indicated allele on a } L E U 2 \text {-marked plasmid and wild-type } S L Y 1 \text { (or } \\
\text { USO1) on a counter-selectable URA3-marked balancer plasmid was assessed following ejection of the balancer } \\
\text { plasmid on 5-FOA plates incubated at } 30^{\circ} \mathrm{C} \text { for } 3 \text { days. } \\
{ }^{2} \text { Growth of } y p t 1-3 \text { cells bearing the indicated alleles of } S L Y 1 \text { on a } L E U 2 \text {-marked plasmid, on SC -Leu media } \\
\text { shifted to non-permissive temperatures }\left(34^{\circ} \text { and } 37^{\circ} \mathrm{C} \text { ) for } 3 \text { days. }\right.\end{array}$} \\
\hline
\end{tabular}




\section{Supplementary Table 4. Yeast strains and plasmids used in this study}

\begin{tabular}{|c|c|c|}
\hline Strain/plasmid ID & Description/genotype & Source/reference \\
\hline \multicolumn{3}{|c|}{ Yeast Strains } \\
\hline AMY2143 (CBY481) & MATalpha ura3-1 trp1-1 ade2-1 leu2-3,112 can1-100 ypt1-3 & C. Barlowe \\
\hline AMY2349 & $\begin{array}{l}\text { MATalpha ura3-1 trp1-1 ade2-1 leu2-3,112 can1-100 ypt1-3 } \\
\text { CBB1395 (URA3 } 2 \mu \text { m YPT1) }\end{array}$ & C. Barlowe \\
\hline AMY2144 (CBY1297) & 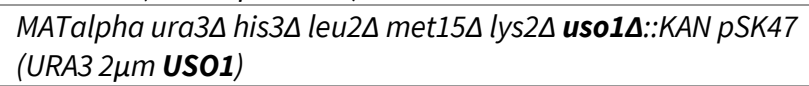 & C. Barlowe \\
\hline AMY2232 (CBY73) & 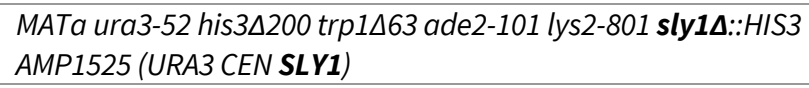 & C. Barlowe \\
\hline AMY2141 (Y8205) & 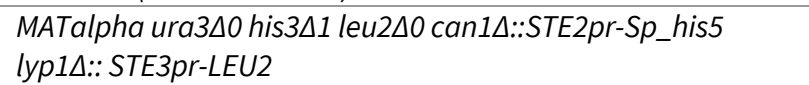 & C. Boone \\
\hline AMY2440 & 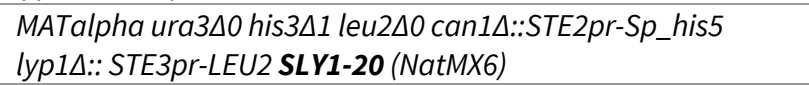 & This study \\
\hline AMY2441 & 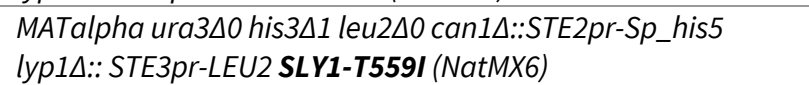 & This study \\
\hline AMY2442 & 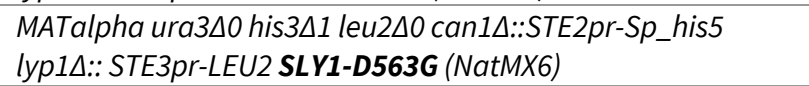 & This study \\
\hline AMY2443 & 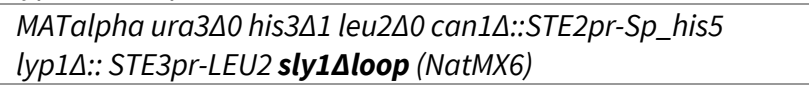 & This study \\
\hline AMY2580 & 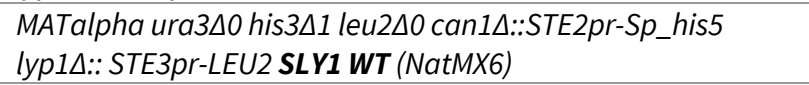 & This study \\
\hline \multicolumn{3}{|c|}{ Yeast expression plasmids } \\
\hline AMP1910 & pRS415::SLY1 & This study \\
\hline AMP1578 & pRS415::SLY1-20 & This study \\
\hline AMP1588 & pRS415::SLY1(T559I) & This study \\
\hline AMP1589 & pRS415::SLY1(D563G) & This study \\
\hline AMP2052 & pRS415::sly1 1 loop & This study \\
\hline AMP1911 & pRS415:: sly1-pa21 & This study \\
\hline AMP1912 & pRS415:: sly1-20-pa21 & This study \\
\hline Various & pRS415::SLY1*1 & This study \\
\hline pSK47 & pRS426::USO1 & C. Barlowe \\
\hline CBB1395 & pRS426::YPT1 & C. Barlowe \\
\hline pDN524 & pRS424 with additional restriction sites flanking $2 \mu$ origin & Lobingier et al., 2014 \\
\hline pDN317 & pDN524::SEC17 & Lobingier et al., 2014 \\
\hline pDN367 & pDN524::sec17-FSMS (F21S, M22S) & This study \\
\hline \multicolumn{3}{|c|}{ E. coli SNARE expression plasmids } \\
\hline AMP1792 & pET-30::His 6 -(3C)-Sed5 & $\begin{array}{l}\text { (Furukawa and Mima, } \\
\text { 2014) }\end{array}$ \\
\hline AMP1961 & pET-30:: His $_{6}-(3 \mathrm{C})-\mathrm{Sed} 5 \mathrm{Habc}(22-210)$ & This study \\
\hline AMP1960 & pET-30:: His $6-(3 \mathrm{C})-\mathrm{Sed} 5 \mathrm{~N}-\mathrm{Habc}(1-210)$ & This study \\
\hline AMP1973 & pET-49::GST- His 6 -(3C)-Sed5cyt(1-319) & This study \\
\hline AMP2020 & pET-30:: His6-(3C)-Bos1(Q153D) & $\begin{array}{l}\text { (Furukawa and Mima, } \\
\text { 2014) but modified }\end{array}$ \\
\hline AMP1794 & pET-30:: His6-(3C)-Bet1 (with corrected missense mutation) & $\begin{array}{l}\text { (Furukawa and Mima, } \\
\text { 2014) but corrected }\end{array}$ \\
\hline AMP1795 & pET-41::GST- His6-(3C)-Sec22 & $\begin{array}{l}\text { (Furukawa and Mima, } \\
\text { 2014) }\end{array}$ \\
\hline \multicolumn{3}{|c|}{ E. coli SNARE chaperone expression plasmids } \\
\hline AMP1547 & pTYB12::intein-CBD-Sec17 & $\begin{array}{l}\text { (Schwartz and Merz, } \\
\text { 2009) }\end{array}$ \\
\hline AMP77 & pQE9:: His 6 -SEC18 & (Haas and Wickner 1996 \\
\hline
\end{tabular}


bioRxiv preprint doi: https://doi.org/10.1101/2020.01.16.906719; this version posted January 16, 2020. The copyright holder for this preprint (which was not certified by peer review) is the author/funder, who has granted bioRxiv a license to display the preprint in perpetuity. It is made available under aCC-BY-NC-ND 4.0 International license.

\begin{tabular}{|c|c|c|}
\hline \multicolumn{3}{|c|}{ E. coli Sly1 expression plasmids } \\
\hline AMP1649 (pBL51) & pHIS-Parallel1:: His - $_{6}$ (TEV)-Sly1 & $\begin{array}{l}\text { (Lobingier and Merz, } \\
\text { 2014) }\end{array}$ \\
\hline AMP1651 & pHIS-Parallel1:: His 6 -(TEV)-Sly1-20 & This study \\
\hline AMP1652 & pHIS-Parallel1:: His6-(TEV)-Sly1(T559I) & This study \\
\hline AMP1653 & pHIS-Parallel1:: His $6_{-}$(TEV)-Sly1(D563G) & This study \\
\hline AMP1654 & 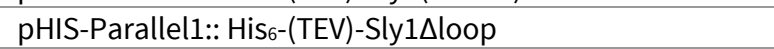 & This study \\
\hline AMP1936 & 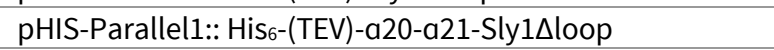 & This study \\
\hline AMP1937 & pHIS-Parallel1:: His $6^{-}$(TEV)-a21-Sly1 $\Delta$ loop & This study \\
\hline AMP1939 & pHIS-Parallel1:: His6-(TEV)-a20-pa21-Sly1 $\Delta$ loop & This study \\
\hline AMP1940 & pHIS-Parallel1:: His 6 -(TEV)-pa21-Sly1 $\Delta$ loop & This study \\
\hline AMP1932 & pHIS-Parallel1:: His ${ }_{6}-(T E V)-S l y 1-p a 21$ & This study \\
\hline AMP1933 & pHIS-Parallel1:: His 6 -(TEV)-Sly1-20-pa21 & This study \\
\hline \multicolumn{3}{|c|}{ E. coli miscellaneous expression plasmids } \\
\hline AMP1881 & pET-49::GST- His6-(3C) & This study \\
\hline AMP2019 & pET-30:: His ${ }_{8}$-HRV3C(Protease) & This study \\
\hline AMP2016 & pET-49::GST- His6-(Thrombin)-HRV3C(Protease) & This study \\
\hline AMP203 & MBP-(TEV)- His $\sigma_{-}$TEV & (Kapust et al., 2002) \\
\hline AMP2018 & pET-28- His $_{6}-\mathrm{CFP}(3 \mathrm{C})$ YFP (for HRV3C protease FRET assay) & This study \\
\hline
\end{tabular}

\begin{tabular}{|c|c|c|c|}
\hline \multicolumn{4}{|c|}{ Supplementary Table 5. SNARE RPL lipid compositions used in this study } \\
\hline \multicolumn{2}{|l|}{ ER mimetic RPLs (R-SNARE) } & \multicolumn{2}{|c|}{ Golgi mimetic RPLs (Qabc-SNARE) } \\
\hline Lipid & mol\% & Lipid & mol\% \\
\hline 18:1 ( $\triangle 9$-Cis) PC & $35.0 \%$ & 18:1 ( $\Delta 9$-Cis) PC & $35.0 \%$ \\
\hline 16:0-18:1 PE & $16.0 \%$ & 16:0-18:1 PE & $14.0 \%$ \\
\hline Soy PI & $6.3 \%$ & Soy PI & $6.3 \%$ \\
\hline 16:0-18:1 PS & $5.6 \%$ & 16:0-18:1 PS & $5.6 \%$ \\
\hline 16:0-18:1 PA & $3.5 \%$ & 16:0-18:1 PA & $3.5 \%$ \\
\hline 18:1 CDP DG & $1.4 \%$ & 18:1 DG & $1.4 \%$ \\
\hline Brain $\mathrm{PI}(4) \mathrm{P}$ & $2.0 \%$ & Brain $\mathrm{PI}(4) \mathrm{P}$ & $2.0 \%$ \\
\hline Ergosterol & $30.0 \%$ & Ergosterol & $30.0 \%$ \\
\hline 16:0 Marina Blue ${ }^{\mathrm{TM}} \mathrm{DHPE}$ & $0.2 \%$ & 16:0 NBD-PE & $2.2 \%$ \\
\hline
\end{tabular}

